\title{
Marine Mollusk Fauna of Kosrae Island, Federated States of Micronesia
}

\author{
Sang-Hwa Lee ${ }^{1}$ and Joong-Ki Park ${ }^{1,2^{*}}$ \\ ${ }^{1}$ Graduate Program in Cellular Biology and Genetics, College of Medicine, Chungbuk National University, Cheongju 361-763, \\ Korea \\ ${ }^{2}$ Department of Parasitology, College of Medicine, Chungbuk National University, Cheongju 361-763, Korea
}

\begin{abstract}
The Federated States of Micronesia consists of four states of Yap, Chuuk, Pohnpei and Kosrae, which are located in the West Pacific Ocean. In order to investigate molluscan fauna of Kosrae Island, field survey was made twice from 21st to 30th of January, 2011 and from 6th to 17th of January, 2012 for four localities including 10 intertidal and 14 subtidal zones of Kosrae Island. The mollusk samples collected were identified based on their morphological characteristics, comprising a total of 120 species from 30 families through this survey. In this study, we provided species list and illustrations for 120 species identified, and combined these with the previous record, resulting in a total of 208 species from 47 families in Kosrae Island.
\end{abstract}

Keywords: Marine Mollusks, Kosrae Island, Micronesia

\section{INTRODUCTION}

The Federated States of Micronesia (FSM) consists of four major islands - Yap, Chuuk, Pohnpei and Kosrae that are located in the West Pacific Ocean. Among these islands Kosrae Island is the smallest one with approximately 112 square kilometers area which was formed by the volcanic activation (Edward, 2002). Kosrae has spacious, well-developed seaward atoll reefs that embrace a rich source of marine biodiversity. About 1,200 mollusks species were estimated to exist in FSM (FSM Department of Economic Affairs, 2002), but a few previous reports has been made by some authorities for molluscan fauna of Micronesia (Demond, 1957; Ladd, 1960). Meanwhile, Micronesian mollusks, particularly gastropods comprising 175 species of 25 families were reported by Demond (1957). Kosrae's

Received: December 12, 2013; Revised: December 20, 2013; Accepted: December 21, 2013

Corresponding author : Joong-Ki Park

Tel: +82 (43) 261-2843 e-mail: jkpyou@chungbuk.ac.kr $1225-3480 / 24504$

This is an Open Access article distributed under the terms of the Creative Commons Attribution Non-Commercial License with permits unrestricted non-commercial use, distribution, and reproducibility in any medium, provided the original work is properly cited. molluscan fauna has never been paid much attention except for Eldredge et al., (1979) that firstly reported 150 species of 42 families including 17 unidentified species. In order to extend investigation of molluscan fauna of Kosrae Island, we conducted field survey for four localities in Kosrae including 10 intertidal and 14 subtidal zones. In this study, we provided species list and illustrations for 120 species identified, and combined these with the previous record of Eldredge et al., (1979).

\section{MATERIALS AND METHODS}

Mollusk samples were collected at intertidal and subtidal zones of Lelu, Malem, Tafunsak, and Utwe from 21st to 30th of January, 2011 and 6th to 17th of January, 2012 (Fig. 1). All collected specimens were preserved in 95\% ethanol solution and then identified based on shell morphological characters. For species identification, we refered to some literatures by taxonomic authorities (Abbott 1961; 1968; 1973; Hinton, 1972; Lamprell and Whitehead, 1992; Lamprell and Healy, 1998; Poppe, 2008a; 2008b; Wilson, 1993; 1994) 


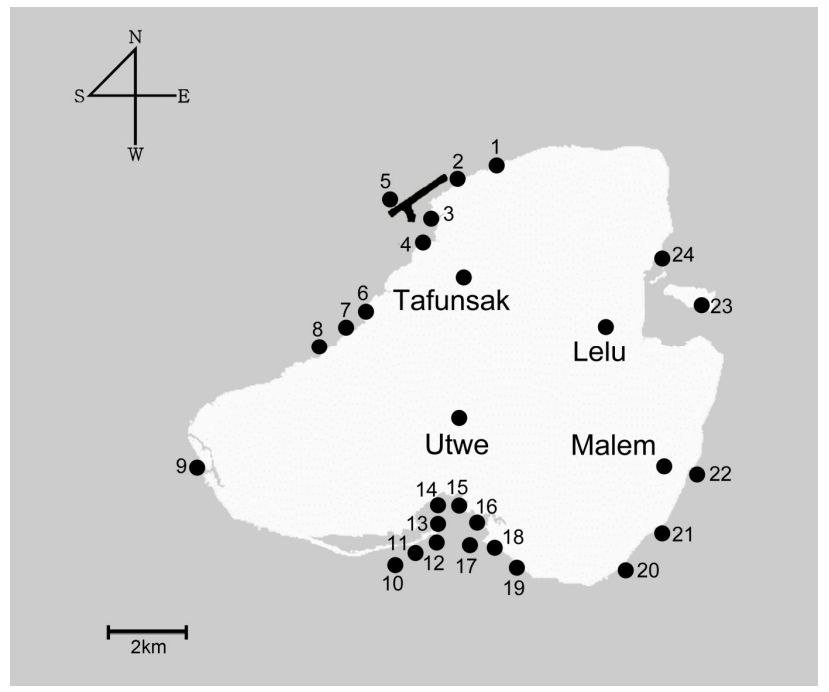

Fig 1. Map showing the Kosrae collection localities. 1, Tafunsak-1 $\left(5^{\circ} 21^{\prime} 55.00^{\prime \prime} \mathrm{N}, 162^{\circ} 58^{\prime} 42.00^{\prime \prime} \mathrm{E}\right)$; 2, Tafunsak-2 ( $\left.5^{\circ} 21^{\prime} 44.00^{\prime \prime} \mathrm{N}, 162^{\circ} 58^{\prime} 01.00 " \mathrm{E}\right)$; 3 , Tafunsak-3 ( $\left.5^{\circ} 21^{\prime} 10.00^{\prime \prime} \mathrm{N}, 162^{\circ} 56^{\prime} 50.00^{\prime \prime} \mathrm{E}\right)$; 4, Tafunsak-4 $\left(5^{\circ} 21^{\prime} 02.00^{\prime \prime} \mathrm{N}, 162^{\circ} 57^{\prime} 37.00^{\prime \prime} \mathrm{E}\right)$; 5, Tafunsak-5 (5 $\left.{ }^{\circ} 21^{\prime} 17.00 " \mathrm{~N}, 162^{\circ} 57^{\prime} 41.00 " \mathrm{E}\right)$; 6, Tafunsak-6 (519'45.54"N, 162 $\left.56^{\circ} 35.76 " \mathrm{E}\right)$; 7, Tafunsak-7 $\left(5^{\circ} 16^{\prime} 25.00^{\prime \prime} \mathrm{N}, 162^{\circ} 57^{\prime} 35.00^{\prime \prime} \mathrm{E}\right)$; 8, Tafunsak-8 $\left(5^{\circ} 19^{\prime} 24.00^{\prime \prime} \mathrm{N}, 162^{\circ} 56^{\prime} 08.00 " \mathrm{E}\right)$; 9, Tafunsak-9 (5॰17'30.84"N, $\left.162^{\circ} 54^{\prime} 03.84 " \mathrm{E}\right)$; 10, Utwe-1 (5॰15'39.10"N, 16257'31.80"E); 11 , Utwe-2 (5'16'12.60"N, $\left.162^{\circ} 58^{\prime} 10.02^{\prime \prime E}\right)$;

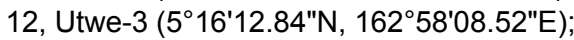
13, Utwe-4 (516'19.32"N, 16257'37.14"E);

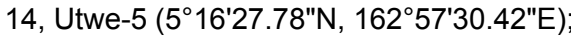

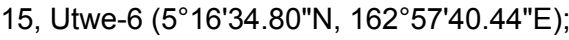
16, Utwe-7 (516'40.02"N, 162 57'33.36"E); 17, Utwe-8 (516'18.00"N, 162 58'28.00"E); 18 , Utwe-9 (5'16'11.60"N, $\left.162^{\circ} 58^{\prime} 27.87 " \mathrm{E}\right)$; 19 , Utwe-10 (5¹5'46.50"N, $\left.162^{\circ} 59^{\prime} 00.66 " \mathrm{E}\right)$; 20, Malem-1 (5॰16'07.74"N, $\left.163^{\circ} 01^{\prime} 00.72 . " \mathrm{E}\right)$; 21, Malem-2 (5'17'16.00"N, $\left.163^{\circ} 01^{\prime} 50.00^{\prime \prime} \mathrm{E}\right)$; 22, Malem-3 (5॰18'04.00"N, $\left.163^{\circ} 01^{\prime} 53.00^{\prime \prime E}\right)$; 23, Lelu-1 (519'58.00"N, 16301'54.00"E); 24 , Lelu-2 ( $\left.5^{\circ} 20^{\prime} 42.00^{\prime \prime} \mathrm{N}, 163^{\circ} 01^{\prime} 23.00 " \mathrm{E}\right)$.

\section{RESULTS AND DISCUSSION}

In the present study, a total of 120 mollusk species were identified from 55 genera of 30 families belong to two classes Gastropoda and Bivalvia. Along with previous record (Eldredge et al., 1979), all molluscan species identified from this study are listed in Table 1. Among 120 identified species in this study, gastropods account for the majority of species count (109 out of 120 species), while the rest (only 11 species) are bivalves. When species abundance was compared at family lavel, two families Muricidae and Conidae are the most common and species-rich among 30 molluscan families found, accounting for $36 \%$ of total species collected (25 muricid species and 18 conid species, respectively). There are some species belong to seven families that are commonly found in all four our survey areas (see Table 1 for more details): Cypraea moneta, Cypraea tigris (Cypraeidae), Trochus niloticus (Trochidae), Cantharus undosus, Engina mendicaria (Buccinidae), Conus ebraeus (Conidae), Mitra chrysalis, Mitra litterata (Mitridae), Morula granulate, Thais tuberosa, Drupa ricinus (Muricidae), Vasum turbinellum (Turbnellidae).

In an earlier report on Kosrae molluscan fauna, Eldredge et al., (1979) identified 150 species of 42 families based on samples collected from Okat area in Kosrae. Of 120 species identified in the present study, 62 species of 18 families were the same species as those reported from Eldredge et al., (1979), but the remaining 58 species of 12 families were newly found from Kosrae Island (Table 1). Combining them together reported from both studies, the number of marine mollusk species in Kosrae Island becomes 208 species from 47 families. The Federated States of Micronesia including Kosrae Island are known to have well-developed coral reefs containing a high species diversity of marine life and relatively less disrupted marine environments (Edward, 2002). On the contrary, the faunal study of Kosrae Island, especially molluscan fauna in this region has not yet been paid much attention, except that of Eldredge et al., (1979). Although the present study adds some updates of molluscan fauna, it is still far from being complete to fully understand magnitude of species diversity in Korae coastal system, requiring further in-depth survey of marine molluscan fauna in this area.

\section{ACKNOWLEDGMENTS}

This work was supported by a grant from Marine Biotechnology Program funded by the Ministry of Oceans and Fisheries for the "Development of overseas marine biological resources and their utilization 
Table 1. List of the marine mollusk species collected from the study of Eldredge et al., (1979) and the present study.

\begin{tabular}{|c|c|c|c|c|c|c|}
\hline \multirow{2}{*}{ Species } & \multirow{2}{*}{$\begin{array}{c}\text { Eldredge et al. } \\
\quad(1979)\end{array}$} & \multicolumn{4}{|c|}{ This study } & \multirow{2}{*}{ Plate } \\
\hline & & Tafunsak & Lelu & Malem & Utwe & \\
\hline \multicolumn{7}{|l|}{ Gastropoda } \\
\hline \multicolumn{7}{|l|}{ Patellidae } \\
\hline Patella flexuosa optima & & + & & + & + & Pl. 1, fig. A \\
\hline Unidentified spp. & + & & & & & \\
\hline \multicolumn{7}{|l|}{ Fissurellidae } \\
\hline Emarginula incisura & & & & & + & Pl. 1, fig. $\mathrm{B}$ \\
\hline Scutus unguis & & & & + & & Pl. 1, fig. C \\
\hline \multicolumn{7}{|l|}{ Trochidae } \\
\hline Euchelus atratus & + & & & & & \\
\hline Monilea philippiana & + & & & & & \\
\hline Tectus pyramis & + & & & & & \\
\hline Trochus conus & & + & + & & & Pl. 2, fig. B \\
\hline Trochus histrio & & + & & + & & Pl. 1, fig. D \\
\hline Trochus incrassatus & + & + & & & & Pl. 1, fig. E \\
\hline Trochus maculatus & & + & & + & + & Pl. 1, fig. F \\
\hline Trochus niloticus & + & + & + & + & + & Pl. 2, fig. A \\
\hline \multicolumn{7}{|l|}{ Neritidae } \\
\hline Nerita albicilla & + & + & + & & & Pl. 2, fig. C \\
\hline Nerita grayana & + & & & & & \\
\hline Nerita plicata & + & + & + & & & Pl. 2, fig. D \\
\hline Nerita polita & + & + & + & & & Pl. 2, fig. E \\
\hline Nerita reticulata & + & + & + & & & Pl. 3, fig. A \\
\hline Nerita undata & & + & & & + & Pl. 3, fig. B \\
\hline Nerita sp. & + & & & & & \\
\hline Neritina bensoni & + & & & & & \\
\hline \multicolumn{7}{|l|}{ Cerithiidae } \\
\hline Cerithium citrinum & & + & & & & Pl. 3, fig. C \\
\hline Cerithium columna & + & + & & & & Pl. 3, fig. D \\
\hline Cerithium echinatum & + & & & & + & Pl. 3, fig. E \\
\hline Cerithium nodulosum & + & + & & & + & Pl. 3, fig. F \\
\hline Cerithium punctatum & & + & & + & & Pl. 4, fig. A \\
\hline Cerithium traillii & & + & & & & Pl. 4, fig. B \\
\hline Cerithium cf. zonatus & + & & & & & \\
\hline Cerithium spp. & + & & & & & \\
\hline Clypeomorus bifasciata & & + & & & & Pl. 4, fig. C \\
\hline Rhinoclavis aspera & + & + & & & & Pl. 4, fig. D \\
\hline
\end{tabular}


Table 1. (Continued)

\begin{tabular}{|c|c|c|c|c|c|c|}
\hline \multirow{2}{*}{ Species } & \multirow{2}{*}{$\begin{array}{c}\text { Eldredge et al. } \\
\text { (1979) }\end{array}$} & \multicolumn{4}{|c|}{ This study } & \multirow{2}{*}{ Plate } \\
\hline & & Tafunsak & Lelu & Malem & Utwe & \\
\hline \multicolumn{7}{|l|}{ Cerithiidae (cont.) } \\
\hline Rhinoclavis sinensis & + & & & & & \\
\hline \multicolumn{7}{|l|}{ Planaxidae } \\
\hline Unidentified sp. & + & & & & & \\
\hline \multicolumn{7}{|l|}{ Cypraeidae } \\
\hline Cypraea annulus & + & + & + & + & & Pl. 4, fig. $\mathrm{F}$ \\
\hline Cypraea arabica & + & + & & + & + & Pl. 4, fig. $\mathrm{E}$ \\
\hline Cypraea caputserpentis & + & & & & + & Pl. 4, fig. G \\
\hline Cypraea carneola & + & & & & & \\
\hline Cypraea depressa & + & & & & + & Pl. 5, fig. A \\
\hline Cypraea erosa & + & + & & & & Pl. 5, fig. B \\
\hline Cypraea globulus & + & & & & & \\
\hline Cypraea helvola & + & & & & & \\
\hline Cypraea isabella & + & + & & & & Pl. 5, fig. C \\
\hline Cypraea lynx & + & & & & & \\
\hline Cypraea moneta & + & + & + & + & + & Pl. 5, fig. D \\
\hline Сypraea poraria & + & & & & & \\
\hline Cypraea teres & & + & & & & Pl. 5, fig. E \\
\hline Cypraea testudinaria & + & & & & & \\
\hline Cypraea tigris & + & + & + & + & + & Pl. 5, fig. $\mathrm{F}$ \\
\hline Cypraea vitellus & + & & & + & & Pl. 5, fig. G \\
\hline Mauritia maculifera & & + & & & & Pl. 6, fig. A \\
\hline \multicolumn{7}{|l|}{ Littorinidae } \\
\hline Littoraria coccinea & + & & + & + & & Pl. 6, fig. B \\
\hline Littoraria scabra & + & & + & + & & Pl. 6, fig. C \\
\hline Littoraria undulata & + & + & & + & & Pl. 6, fig. D \\
\hline Littorina sp. & + & & & & & \\
\hline Nodolittorina millegrana & + & & & & & \\
\hline Tectarius sp. & & & & & & \\
\hline \multicolumn{7}{|l|}{ Strombidae } \\
\hline Lambis lambis & + & + & & & + & Pl. 6, fig. E \\
\hline Lambis truncata & & & & & + & Pl. 7, fig. A \\
\hline Strombus gibberulus & + & + & + & & & Pl. 7, fig. $\mathrm{B}$ \\
\hline Strombus luhuanus & + & & & & & \\
\hline Strombus mutabilis & + & + & + & & & Pl. 7, fig. C \\
\hline
\end{tabular}


Table 1. (Continued)

\begin{tabular}{ccccccc}
\hline \multirow{2}{*}{ Species } & Eldredge et al. & \multicolumn{4}{c}{ This study } \\
\cline { 3 - 5 } & $(1979)$ & Tafunsak & Lelu & Malem & Utwe & Plate \\
\hline
\end{tabular}

Capulidae

Capulis danieli

Capulis sp.

Calyptraeidae

Cheilea equestris

Naticidae

Natica gualtieriana

Natica zonalus

Polinices melanostomus

Polinices tumidus

Bursidae

Bursa bufonia

Bursa cruentata

Bursa granularis

Tonnidae

\section{Tonna perdix}

Personidae

Distorsio anus

Ranellidae

Charonia tritonis

Cymatium mundum

Cymatium muricinum

Cymatium nicobaricum

Gyrineum natator

Hipponicidae

Hipponix conicus

Buccinidae

Cantharus fumosus

Cantharus undosus

Engina alveolata

Engina mendicaria

Engina sp.

Columbellidae
$+$

$+$

$+$

$+$

$+$

$+$

$+$

$+$

$+$

$+$

$+\quad$ Pl. 8, fig. A

Pl. 8, fig. B
$+$

Pl. 8, fig. C

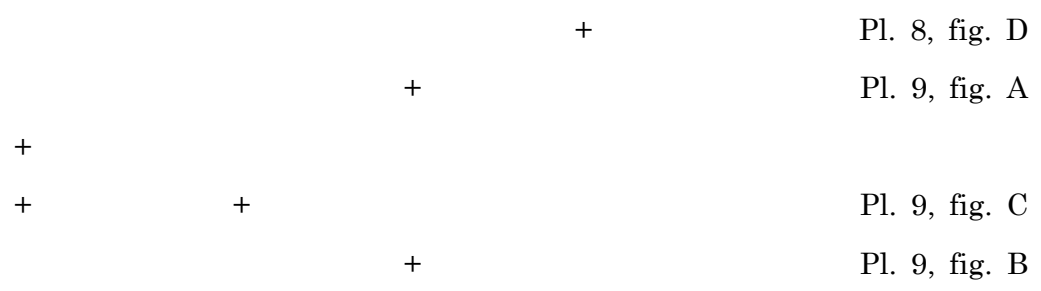

$+$

$+$

Pl. 9, fig. D

$+$

$+$

$+$

$+$

$+$

$+$

$+$

$+\quad+$

$+$

Pl. 9, fig. I 
Table 1. (Continued)

\begin{tabular}{|c|c|c|c|c|c|c|}
\hline \multirow{2}{*}{ Species } & \multirow{2}{*}{$\begin{array}{l}\text { Eldredge et al. } \\
\text { (1979) }\end{array}$} & \multicolumn{4}{|c|}{ This study } & \multirow{2}{*}{ Plate } \\
\hline & & Tafunsak & Lelu & Malem & Utwe & \\
\hline \multicolumn{7}{|l|}{ Fasciolariidae } \\
\hline Latirolagena smaragdula & & + & & & + & Pl. 10, fig. A \\
\hline Latirus sp. & + & & & & & \\
\hline Peristernia sp. & + & & & & & \\
\hline \multicolumn{7}{|l|}{ Nassariidae } \\
\hline Nassarius albescens & + & & & & & \\
\hline Nassarius margaritifer & + & + & & & + & Pl. 10, fig. C \\
\hline Nassarius papillosus & + & & & & + & Pl. 10, fig. B \\
\hline Nassarius vitiensis & + & & & & & \\
\hline \multicolumn{7}{|l|}{ Muricidae } \\
\hline Chicoreus brunneus & & & & & + & Pl. 10, fig. D \\
\hline Coralliophila pyriformis & + & & & & & \\
\hline Coralliophila radula & & & & & + & Pl. 11, fig. A \\
\hline Coralliophila squamulosa & & + & & + & + & Pl. 11, fig. B \\
\hline Coralliophila violacea & + & + & & + & + & Pl. 11, fig. C \\
\hline Cronia margariticola & + & + & + & & & Pl. 11, fig. D \\
\hline Drupa morum & + & & & + & + & Pl. 11, fig. E \\
\hline Drupa ricinus & + & + & + & + & + & Pl. 11, fig. $\mathrm{F}$ \\
\hline Drupa rubusidaeus & + & + & + & & + & Pl. 11, fig. G \\
\hline Drupella cornus & + & + & & & + & Pl. 12, fig. A \\
\hline Drupella rugosa & & + & + & & & Pl. 12, fig. $\mathrm{B}$ \\
\hline Drupina grossularia & & + & & + & + & Pl. 12, fig. C \\
\hline Habromorula ambrosia & & & + & & & Pl. 12, fig. D \\
\hline Leptoconchus lamarckii & + & & & & & \\
\hline Leptoconchus striatus & + & & & & & \\
\hline Mancinella hippocastanus & + & & + & & & Pl. 12, fig. $\mathrm{F}$ \\
\hline Morula biconica & + & & & & & \\
\hline Morula granulate & + & + & + & + & + & Pl. 12, fig. E \\
\hline Morula marginalba & & + & + & & & Pl. 12, fig. G \\
\hline Morula nodicostata & & + & + & & & Pl. 12, fig. $\mathrm{H}$ \\
\hline Morula uva & + & + & + & + & & Pl. 12, fig. I \\
\hline Morula sp. & + & & & & & \\
\hline
\end{tabular}


Table 1. (Continued)

\begin{tabular}{|c|c|c|c|c|c|c|}
\hline \multirow{2}{*}{ Species } & \multirow{2}{*}{$\begin{array}{c}\text { Eldredge et al. } \\
\quad(1979)\end{array}$} & \multicolumn{4}{|c|}{ This study } & \multirow{2}{*}{ Plate } \\
\hline & & Tafunsak & Lelu & Malem & Utwe & \\
\hline \multicolumn{7}{|l|}{ Muricidae (cont.) } \\
\hline Muricodrupa fenestrata & & + & + & & + & Pl. 13, fig. A \\
\hline Nassa serta & + & & & & & \\
\hline Nassa francolina & & + & & & & Pl. 13, fig. C \\
\hline Rapa bulbiformis & & + & & & & Pl. 13, fig. D \\
\hline Rapa rapa & + & & & + & & Pl. 13, fig. E \\
\hline Semiricinula marginatra & & + & & & & Pl. 13, fig. B \\
\hline Thais aculeata & & & + & & & Pl. 14, fig. A \\
\hline Thais armigera & & & & + & + & Pl. 14, fig. B \\
\hline Thais tuberosa & & + & + & + & + & Pl. 15, fig. A \\
\hline Quoyula monodonta & + & & & & & \\
\hline \multicolumn{7}{|l|}{ Harpidae } \\
\hline Harpa amouretta & + & & & & & \\
\hline \multicolumn{7}{|l|}{ Mitridae } \\
\hline Imbricaris punctata & + & & & & & \\
\hline Mitra acuminata & + & + & & + & + & Pl. 15, fig. B \\
\hline Mitra auriculoides & & & & & + & Pl. 15, fig. C \\
\hline Mitra chrysalis & & + & + & + & + & Pl. 15, fig. D \\
\hline Mitra decurtata & & & & + & + & Pl. 15, fig. E \\
\hline Mitra eremitarum & + & + & & & & Pl. 15, fig. $\mathrm{F}$ \\
\hline Mitra litterata & & + & + & + & + & Pl. 16, fig. A \\
\hline Mitra mitra & + & & & & & \\
\hline Mitra paupercula & + & + & + & + & & Pl. 16, fig. B \\
\hline Mitra stictica & + & & & & & \\
\hline Vexillum plicarium & + & & & & & \\
\hline \multicolumn{7}{|l|}{ Turbinidae } \\
\hline Astralium rhodostoma & + & & & & + & Pl. 16, fig. C \\
\hline Turbo argyrostomus & + & & & & + & Pl. 16, fig. D \\
\hline Turbo petholatus & + & & & & & \\
\hline \multicolumn{7}{|l|}{ Turbinellidae } \\
\hline Vasum turbinellum & + & + & + & + & + & Pl. 17, fig. A \\
\hline \multicolumn{7}{|l|}{ Conidae } \\
\hline Conus capitaneus & + & & & & & \\
\hline Conus catus & + & & & & & \\
\hline Conus chaldaeus & + & + & & + & + & Pl. 17, fig. B \\
\hline
\end{tabular}


Table 1. (Continued)

\begin{tabular}{|c|c|c|c|c|c|c|}
\hline \multirow{2}{*}{ Species } & \multirow{2}{*}{$\begin{array}{l}\text { Eldredge et al. } \\
\quad(1979)\end{array}$} & \multicolumn{4}{|c|}{ This study } & \multirow{2}{*}{ Plate } \\
\hline & & Tafunsak & Lelu & Malem & Utwe & \\
\hline \multicolumn{7}{|l|}{ Conidae (cont.) } \\
\hline Conus coronatus & + & + & & & & Pl. 17, fig. D \\
\hline Conus distans & + & & & + & & Pl. 17, fig. C \\
\hline Conus ebraeus & + & + & + & + & + & Pl. 17, fig. E \\
\hline Conus eburneus & + & & & & & \\
\hline Conus flavidus & + & & & & & \\
\hline Conus geographus & + & & & & & \\
\hline Conus giorossi & & + & & & & Pl. 17, fig. F \\
\hline Conus leopardus & & + & & & & Pl. 18, fig. A \\
\hline Conus litoglyphus & + & & & & & \\
\hline Conus litteratus & + & & & & & \\
\hline Conus lividus & + & & & & & \\
\hline Conus magus & + & & & & & \\
\hline Conus marmoreus & + & + & & & & Pl. 18, fig. B \\
\hline Conus miles & + & + & & & + & Pl. 18, fig. C \\
\hline Conus miliaris & + & + & & + & + & Pl. 18, fig. D \\
\hline Conus moncuri & & & & & + & Pl. 18, fig. E \\
\hline Conus moreleti & & + & & & & Pl. 18, fig. F \\
\hline Conus musicus & + & & & & & \\
\hline Conus pulicarius & + & + & & & & Pl. 19, fig. A \\
\hline Conus rattus & + & & & & & \\
\hline Conus sanguinolentus & + & + & + & & & Pl. 19, fig. B \\
\hline Conus sponsalis & + & + & & + & + & Pl. 19, fig. C \\
\hline Conus striatus & & + & & & & Pl. 19, fig. D \\
\hline Conus tulipa & & + & + & + & & Pl. 19, fig. E \\
\hline Conus vexillum & + & & & & & \\
\hline Conus vitulinus & & & & & + & Pl. 20, fig. A \\
\hline \multicolumn{7}{|l|}{ Terebridae } \\
\hline Terebra affinis & + & & & & & \\
\hline Terebra babylonia & + & & & & & \\
\hline Terebra columellaris & + & & & & & \\
\hline Terebra crenulata & + & & & & & \\
\hline Terebra feline & + & + & & & & Pl. 20, fig. B \\
\hline Terebra maculate & + & & & & & \\
\hline Terebra paucistriata & + & & & & & \\
\hline
\end{tabular}


Table 1. (Continued)

\begin{tabular}{|c|c|c|c|c|c|c|}
\hline \multirow{2}{*}{ Species } & \multirow{2}{*}{$\begin{array}{l}\text { Eldredge et al. } \\
\quad \text { (1979) }\end{array}$} & \multicolumn{4}{|c|}{ This study } & \multirow{2}{*}{ Plate } \\
\hline & & Tafunsak & Lelu & Malem & Utwe & \\
\hline \multicolumn{7}{|l|}{ Terebridae (cont.) } \\
\hline Terebra subulata & + & & & & & \\
\hline \multicolumn{7}{|l|}{ Janthinidae } \\
\hline Janthina sp. & + & & & & & \\
\hline \multicolumn{7}{|l|}{ Pyramidellidae } \\
\hline Pyrmidella acus & + & & & & & \\
\hline Pyramidella terebellum & + & & & & & \\
\hline \multicolumn{7}{|l|}{ Hydatinidae } \\
\hline Hydatina physis & + & & & & & \\
\hline \multicolumn{7}{|l|}{ Bullidae } \\
\hline Bulla vernicosa & + & & & & & \\
\hline \multicolumn{7}{|l|}{ Aplysiidae } \\
\hline Dolabrifera dolabrifera & + & & & & & \\
\hline Phyllaplysia taylori & + & & & & & \\
\hline \multicolumn{7}{|l|}{ Phyllidiidae } \\
\hline Phyllidia elegans & + & & & & & \\
\hline Phyllidia trilineata & + & & & & & \\
\hline Phyllidia $c f$. variavilis & + & & & & & \\
\hline Phyllidia cf. tula & + & & & & & \\
\hline Phyllidiella pustulosa & & & & & + & Pl. 20, fig. C \\
\hline \multicolumn{7}{|l|}{ Ellobiidae } \\
\hline Melampus flavus & + & & & & & \\
\hline \multicolumn{7}{|l|}{ Bivalvia } \\
\hline \multicolumn{7}{|l|}{ Arcidae } \\
\hline Arca avellana & & & & + & + & Pl. 21, fig. A \\
\hline Arca sp. & + & & & & & \\
\hline Barbatia coma & & & & + & & Pl. 21, fig. B \\
\hline Barbatia foliata & & & & & + & Pl. 21, fig. C \\
\hline \multicolumn{7}{|l|}{ Mytilidae } \\
\hline Modiolus sp. & + & & & & & \\
\hline \multicolumn{7}{|l|}{ Pteriidae } \\
\hline Pinctata margaritafera & + & & & & & \\
\hline \multicolumn{7}{|l|}{ Isognomonidae } \\
\hline Isognomon perna & & + & & + & & Pl. 21, fig. D \\
\hline \multicolumn{7}{|l|}{ Limidae } \\
\hline Ctenoides lischkei & & & & + & + & Pl. 22, fig. A \\
\hline
\end{tabular}


Table 1. (Continued)

\begin{tabular}{|c|c|c|c|c|c|c|}
\hline \multirow{2}{*}{ Species } & \multirow{2}{*}{$\begin{array}{c}\text { Eldredge et al. } \\
\text { (1979) }\end{array}$} & \multicolumn{4}{|c|}{ This study } & \multirow{2}{*}{ Plate } \\
\hline & & Tafunsak & Lelu & Malem & Utwe & \\
\hline \multicolumn{7}{|l|}{ Limidae (cont.) } \\
\hline Lima lima vulgaris & & & & & + & Pl. 22, fig. B \\
\hline \multicolumn{7}{|l|}{ Spondylidae } \\
\hline Spondylus sp. & + & & & & & \\
\hline \multicolumn{7}{|l|}{ Gryphaeidae } \\
\hline Hyotissa hyotis & & + & & & & Pl. 22, fig. C \\
\hline \multicolumn{7}{|l|}{ Ostreidae } \\
\hline Lopha cristagalli & & + & & & + & Pl. 23, fig. A \\
\hline \multicolumn{7}{|l|}{ Cardiidae } \\
\hline Fragrum fragrum & + & & & & & \\
\hline \multicolumn{7}{|l|}{ Chamidae } \\
\hline Chama iostoma & + & & & & & \\
\hline Unidentified sp. & + & & & & & \\
\hline \multicolumn{7}{|l|}{ Tridacnidae } \\
\hline Tridacna maxima & + & + & & & + & Pl. 23, fig. B \\
\hline \multicolumn{7}{|l|}{ Veneridae } \\
\hline Antigona puerpera & & + & & & & Pl. 23, fig. C \\
\hline Gafrarium menkei & & + & & & & Pl. 23, fig. D \\
\hline Pitar sp. & + & & & & & \\
\hline
\end{tabular}

system (PM57121)" and "Marine Mollusk Resource Bank of Korea (MMRBK)".

\section{REFERENCES}

Abbott, R.T. (1959) Indo-Pacific Mollusca, Vol. 1, The department of mollusks. pp. 1-490, Academy of Natural Science of Philadelphia, Philadelphia.

Abbott, R.T. (1968) Indo-Pacific Mollusca, Vol. 2, The department of mollusks. pp. 1-534, Academy of Natural Science of Philadelphia, Philadelphia.

Abbott, R.T. (1973) Indo-Pacific Mollusca, Vol. 3, The department of mollusks. pp. 1-74, Academy of Natural Science of Philadelphia, Philadelphia.

Demond, J. (1957) Micronesian reef-associated gastropods. Pacific Science, 25: 275-341.

Edward, A. (2002) Marine biodiversity of the Federated States of Micronesia. FMS National Bioldiversity Strategy and Action Plan Project.

Eldredge, L.G., Best, B.R., Chernin, M.I., Kropp, R.K., Myers, R.F., and Smalley, T.L. (1979) Marine environmental survey of Okat, Kosrae. University of Guam marine laboratory technical report, 63: 1-101.
FSM (Federated States of Micronesia) Department of Economic Affairs (2002) The Federated States of Micronesia preliminary national report to the secretariat of the convention to combat desertification.

Hinton, A. (1972) Shells of New Guinea and the Central Indo-Pacific. pp. 1-94, Robert Brown and Associates Pty. Ltd., Port Moresby.

Lamprell, K. and Whitehead, T. (1992) Bivalves of Australia. Vol. 1. pp. 1-182, Crawford House Press Pty, Bathurst.

Lamprell, K. and Healy, J. (1998) Bivalves of Australia. Vol. 2. pp. 1-288, Backuys publishers, Bathurst.

Ladd, H.S. (1960) Origin of the pacific island molluscan fauna. American journal of science, 258a: 137-150.

Poppe, G.T. (2008a) Philippin marine mollusks. Vol. 1. Gatropoda, Part 1. pp. 1-758, ConchBooks Co., Hackenheim.

Poppe, G.T. (2008b). Philippin marine mollusks. Vol. 2. Gatropoda, Part 2. pp. 1-848, ConchBooks Co., Hackenheim.

Wilson, B. (1993) Australian marine shells. Vol. 1. Prosobranch gastropods, part 1. pp. 1-408, Odyssey publishing Co., Kallaroo. 
Korean J. Malacol. 29(4): 343-376 2013

Wilson, B. (1994) Australian marine shells. Vol. 2.

Prosobranch gastropods, part 2 (Neogastropods). pp.

1-370, Odyssey Publishing Co., Kallaroo. 

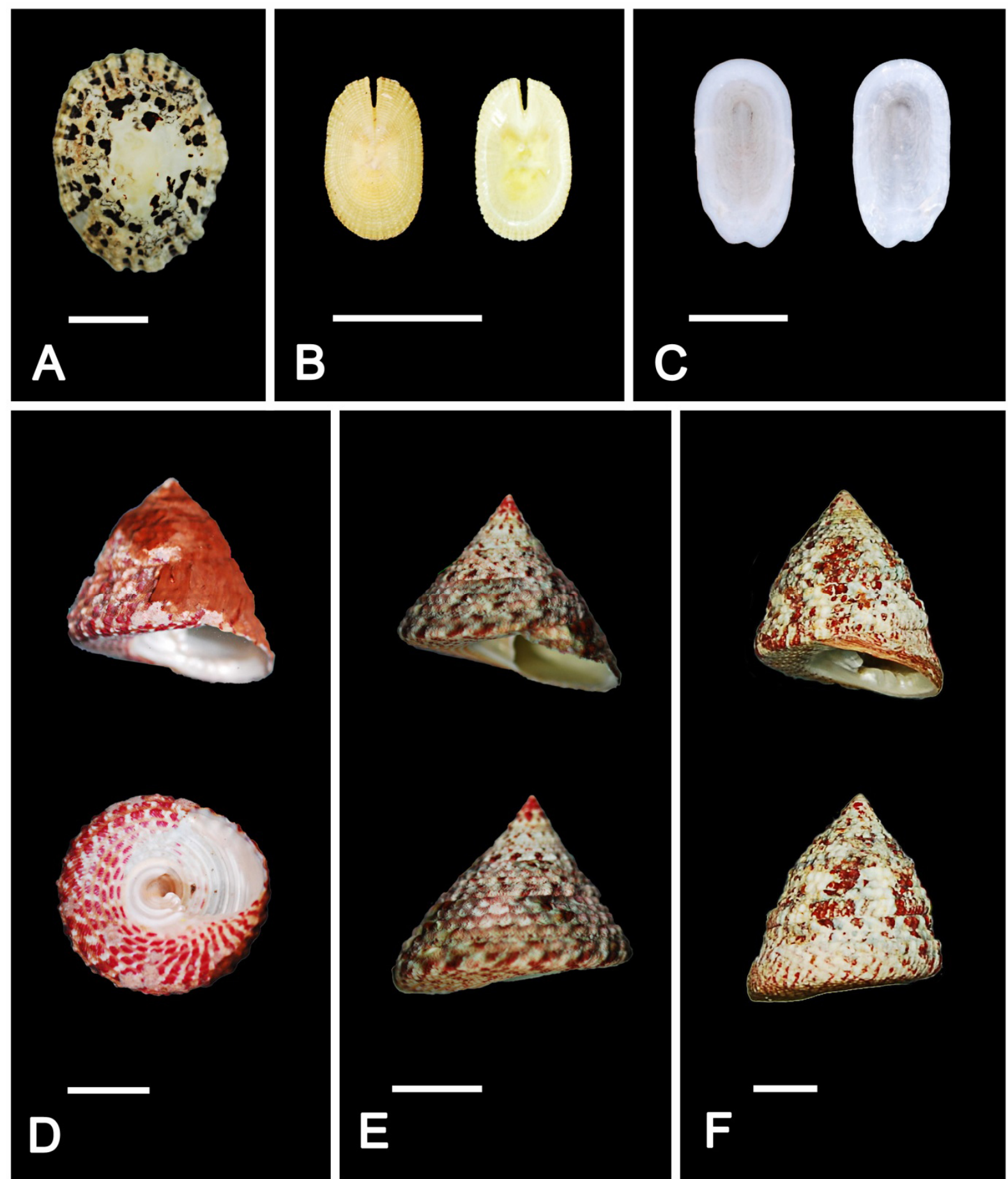

Plate 1: A, Patella flexuosa optima (scale bar = $10 \mathrm{~mm}$ ); B, Emarginula incisura (scale bar = $10 \mathrm{~mm}$ ); C, Scutus unguis (scale bar = $10 \mathrm{~mm}$ ); $\mathbf{D}$, Trochus histrio (scale bar = $10 \mathrm{~mm}$ ); E, Trochus incrassatus (scale bar $=10 \mathrm{~mm}$ ); $\mathbf{F}$, Trochus maculatus (scale bar $=10 \mathrm{~mm}$ ). 

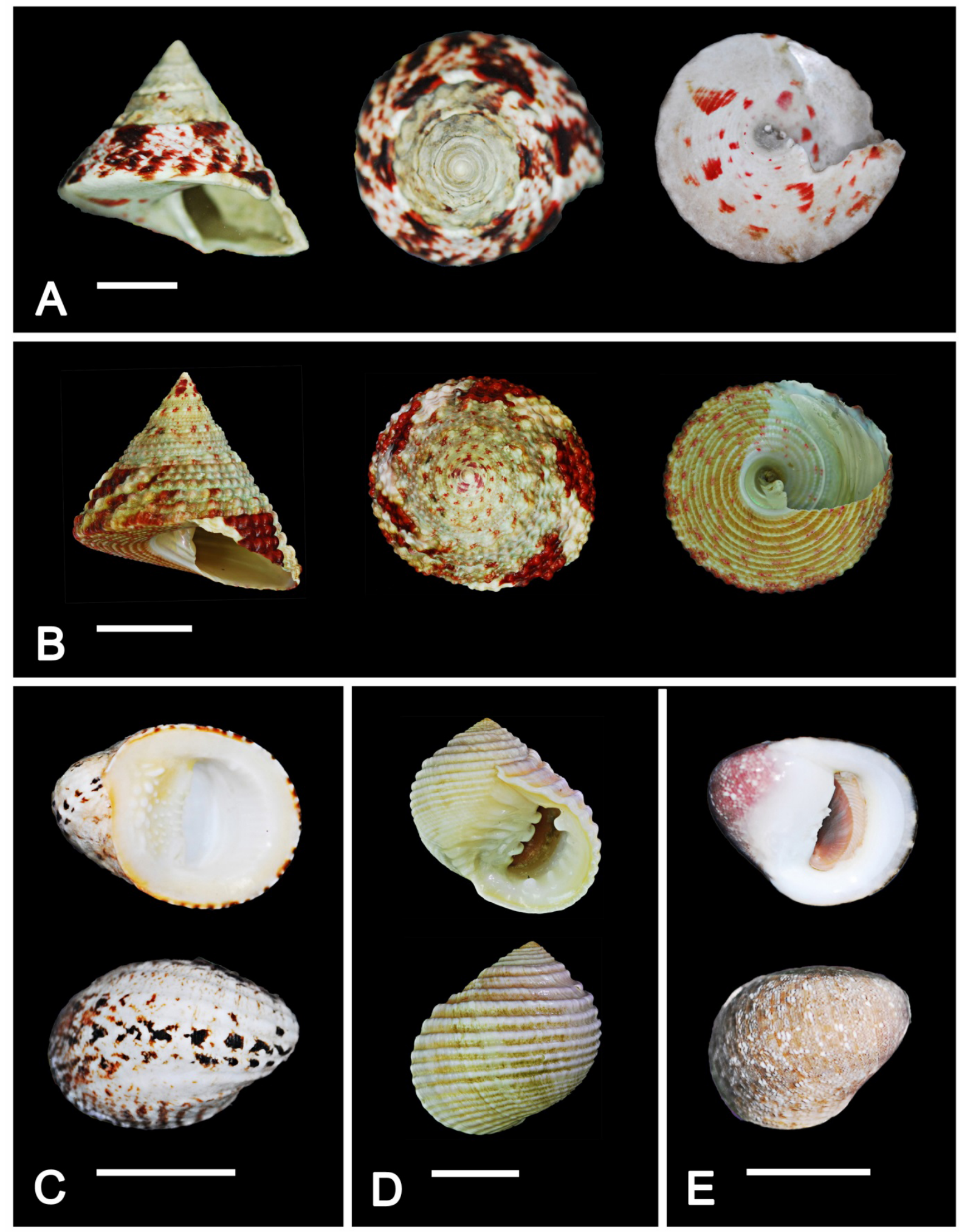

Plate 2: A, Trochus niloticus (scale bar $=10 \mathrm{~mm}$ ); B, Trochus conus $($ scale bar $=10 \mathrm{~mm}) ; \mathbf{C}$, Nerita albicilla $(\mathrm{scale}$ bar $=$ $10 \mathrm{~mm}$ ); D, Nerita plicata (scale bar $=10 \mathrm{~mm}$ ); E, Nerita polita (scale bar $=10 \mathrm{~mm}$ ). 

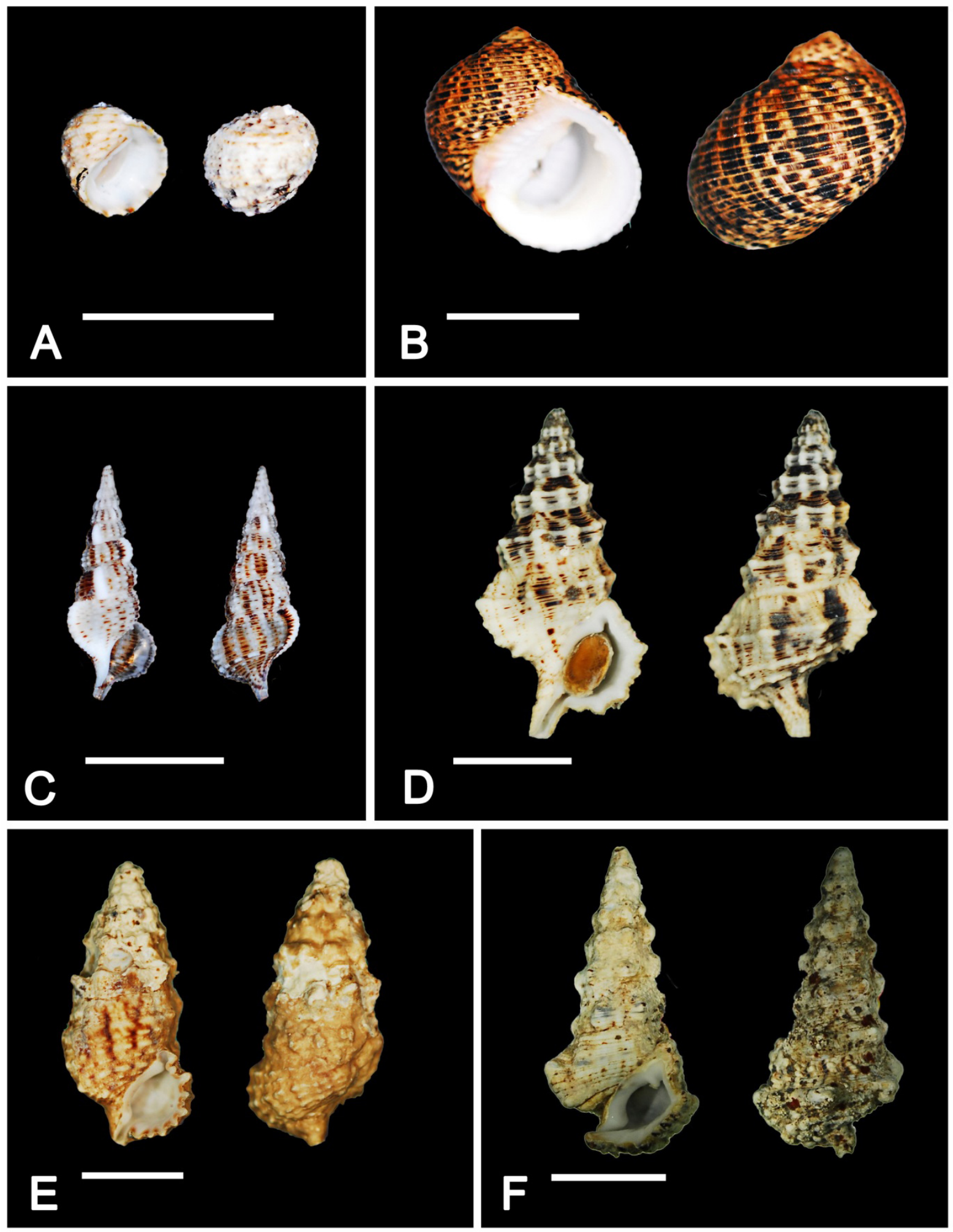

Plate 3: A, Nerita reticulate (scale bar $=10 \mathrm{~mm}$ ); B, Nerita undata (scale bar $=10 \mathrm{~mm}$ ); C, Cerithium citrinum (scale bar $=10 \mathrm{~mm}$ ); $\mathbf{D}$, Cerithium columna (scale bar = $10 \mathrm{~mm}$ ); $\mathbf{E}$, Cerithium echinatum (scale bar $=10 \mathrm{~mm}$ ); $\mathbf{F}$, Cerithium nodulosum (scale bar $=35 \mathrm{~mm})$. 

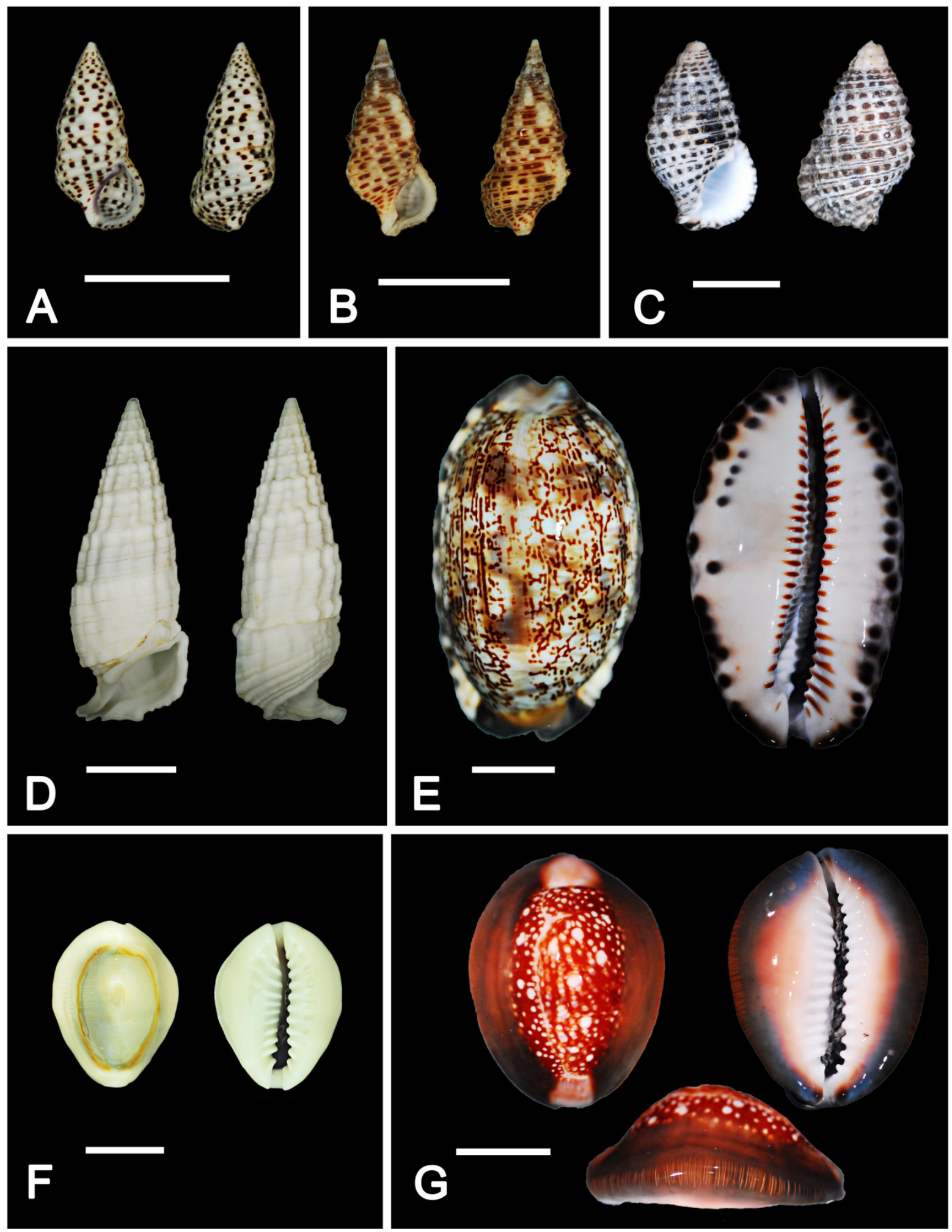

Plate 4: A, Cerithium punctatum (scale bar $=10 \mathrm{~mm}$ ); B, Cerithium traillii (scale bar $=10 \mathrm{~mm}$ ); C Clypeomorus bifasciata (scale bar $=10 \mathrm{~mm}$ ); $\mathbf{D}$, Rhinoclavis aspera (scale bar $=10 \mathrm{~mm}$ ); E, Cypraea Arabica $($ scale bar $=10$ $\mathrm{mm}$ ); F, Cypraea annulus (scale bar $=10 \mathrm{~mm}$ ); G, Cypraea caputserpentis (scale bar $=10 \mathrm{~mm}$ ). 

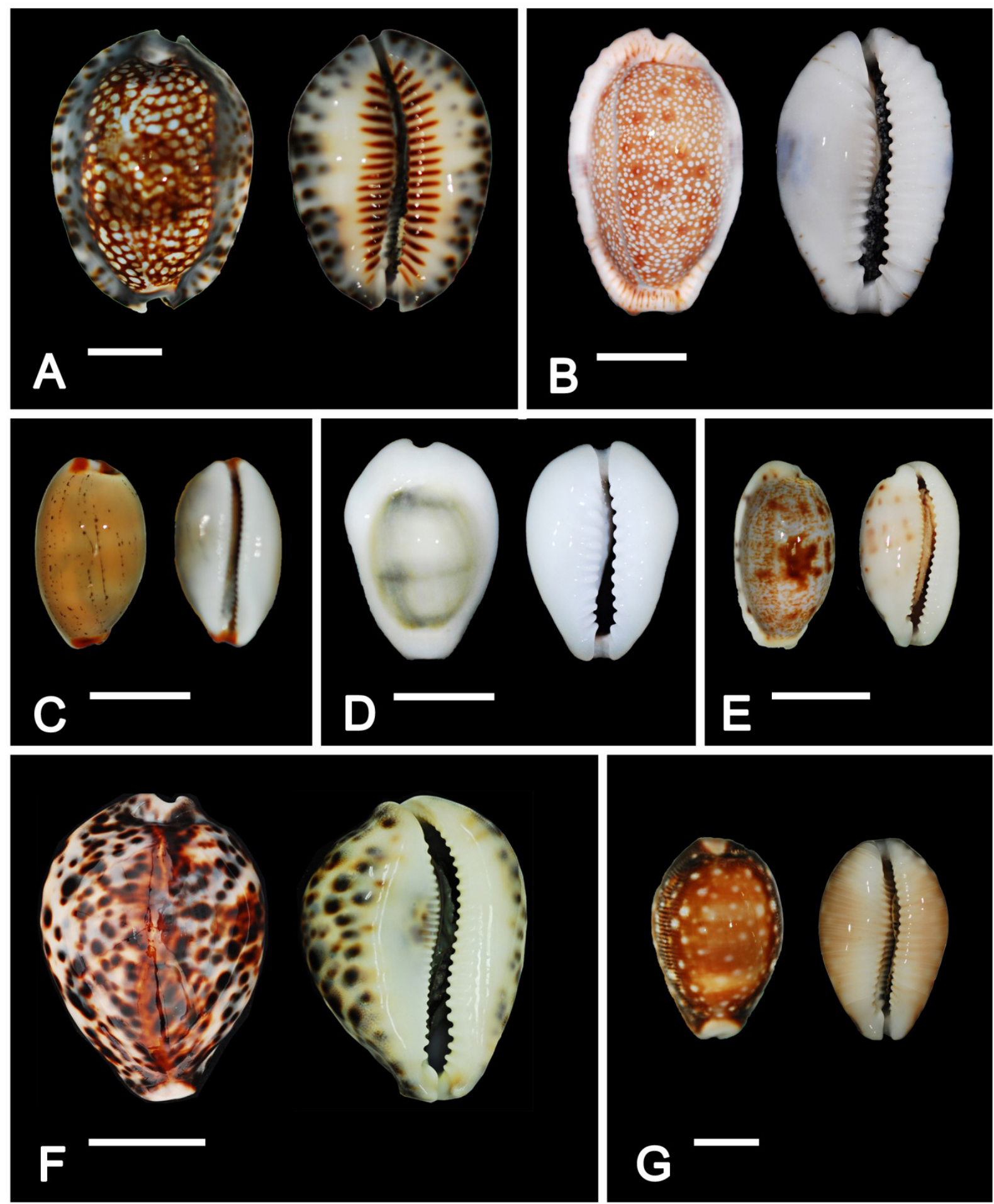

Plate 5: A, Cypraea depressa (scale bar = $10 \mathrm{~mm}$ ); B, Cypraea erosa (scale bar = $10 \mathrm{~mm}$ ); C, Cypraea Isabella (scale bar = $10 \mathrm{~mm}$ ); D, Cypraea moneta (scale bar = $10 \mathrm{~mm}$ ); E, Cypraea teres (scale bar = $10 \mathrm{~mm}$ ); F, Cypraea tigris (scale bar $=30 \mathrm{~mm}$ ); G, Cypraea vitellus (scale bar $=10 \mathrm{~mm}$ ). 

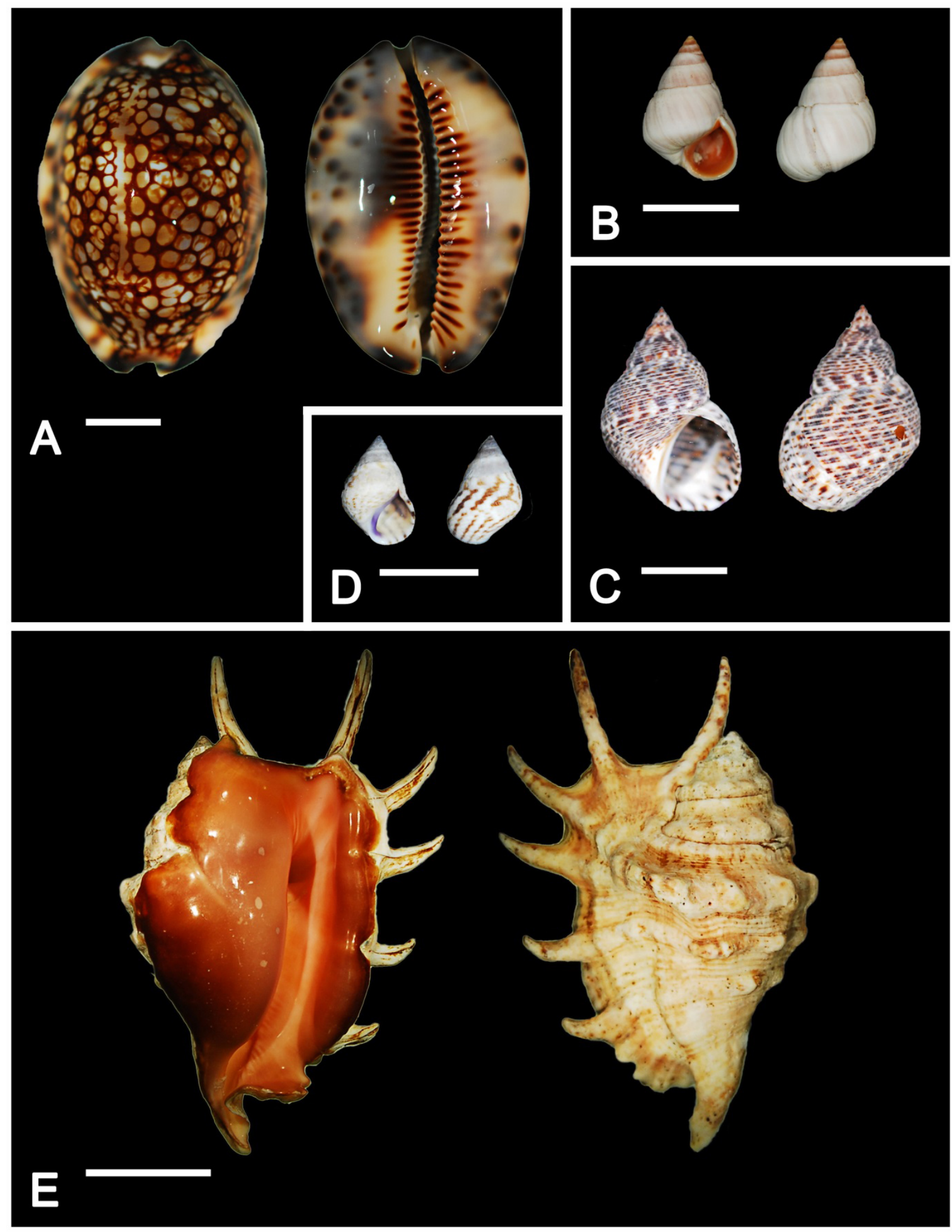

Plate 6: A, Mauritia maculifera (scale bar = $10 \mathrm{~mm}$ ); B, Littoraria coccinea (scale bar = $10 \mathrm{~mm}$ ); C, Littoraria scabra (scale bar =10 mm); D, Littoraria undulate (scale bar =10 mm); E, Lambis lambis (scale bar = $30 \mathrm{~mm}$ ). 

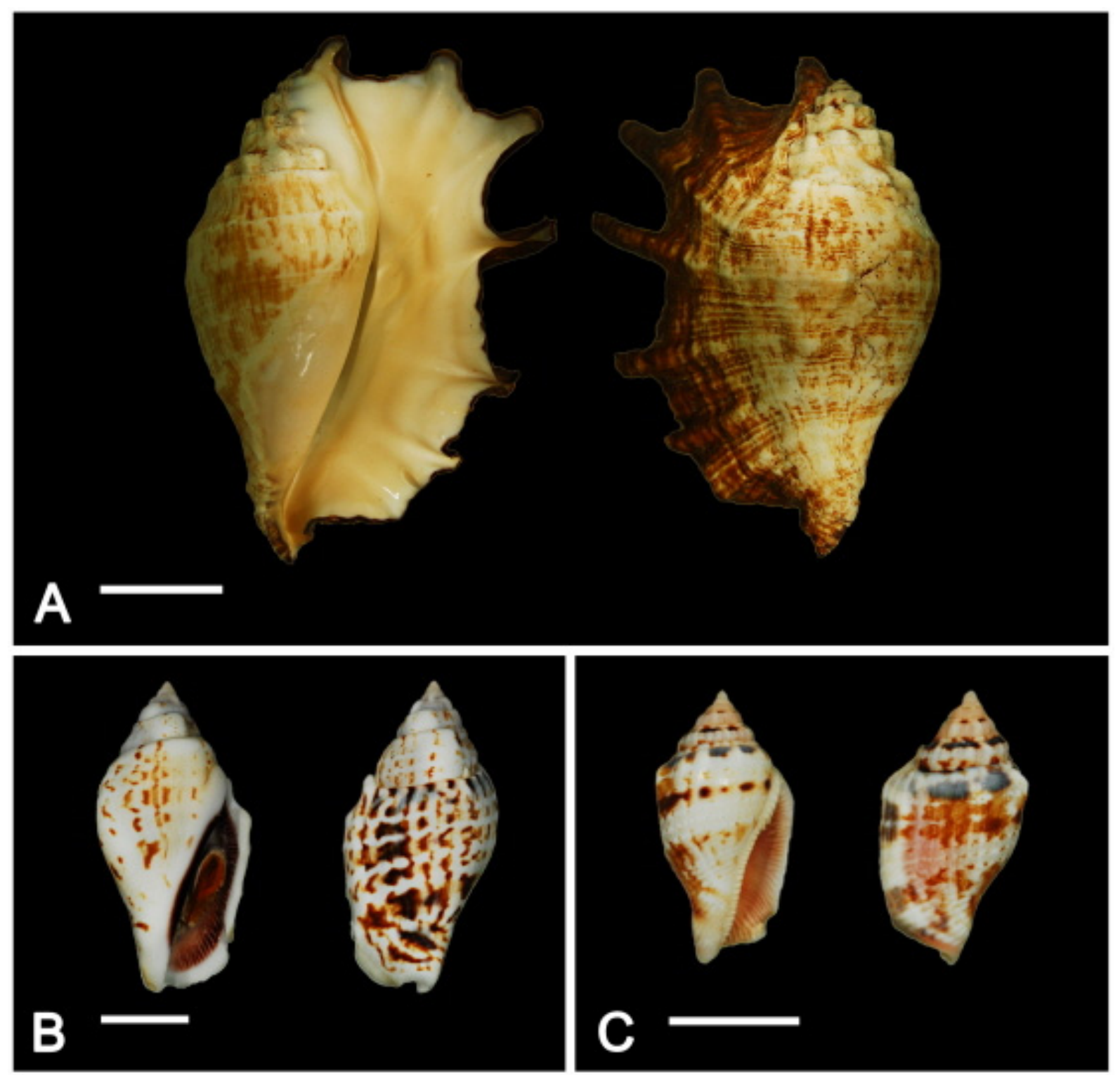

Plate 7: A, Lambis truncate (scale bar $=50 \mathrm{~mm}$ ); B, Strombus gibberulus (scale bar $=10 \mathrm{~mm}$ ); C, Strombus mutabilis $($ scale bar $=10 \mathrm{~mm})$. 

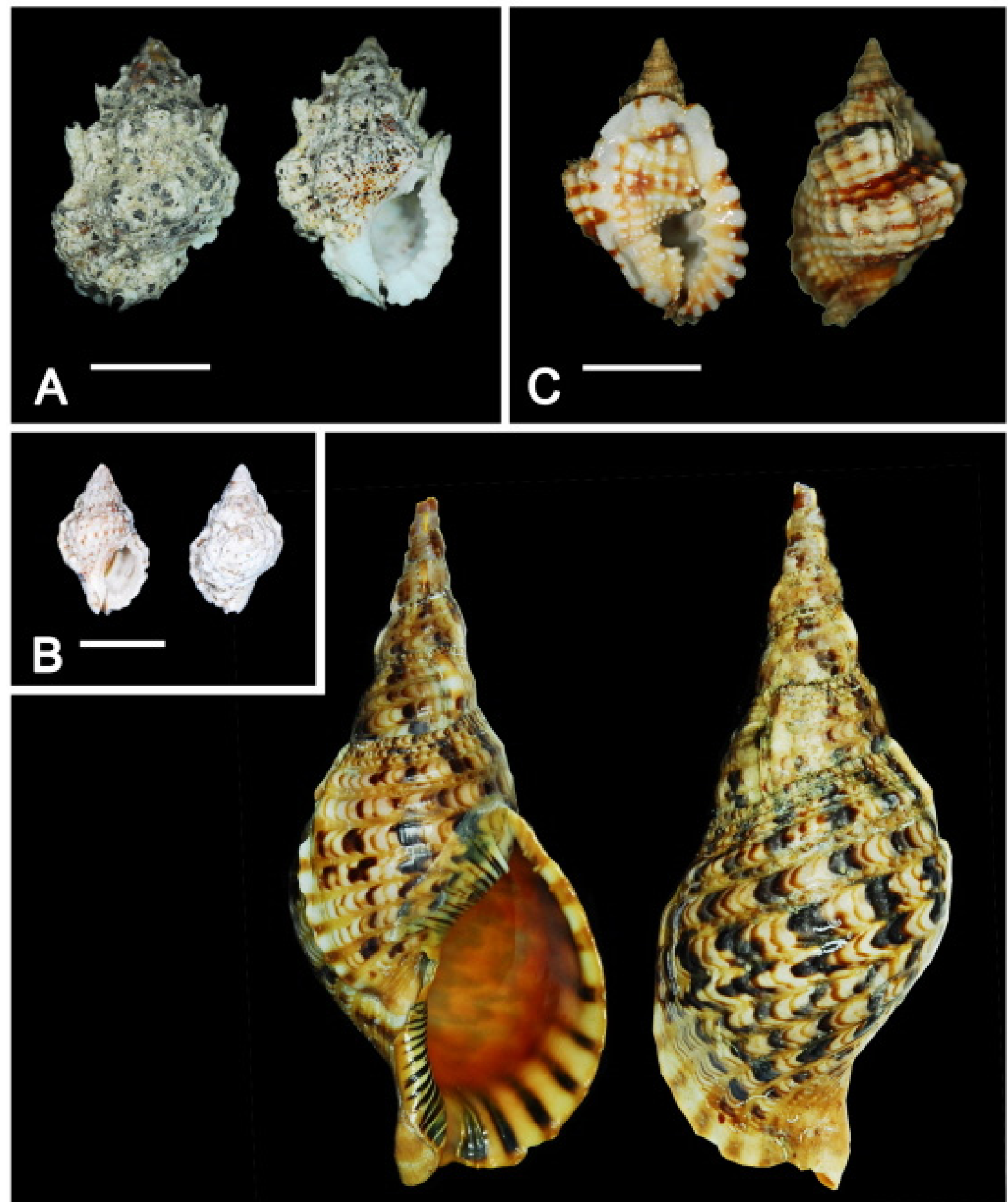

D

Plate 8: A, Bursa bufonia (scale bar = $20 \mathrm{~mm}$ ); B, Bursa granularis (scale bar = $10 \mathrm{~mm}$ ); C, Distorsio anus (scale bar = $20 \mathrm{~mm}$ ); $\mathbf{D}$, Charonia tritonis (scale bar $=100 \mathrm{~mm}$ ). 

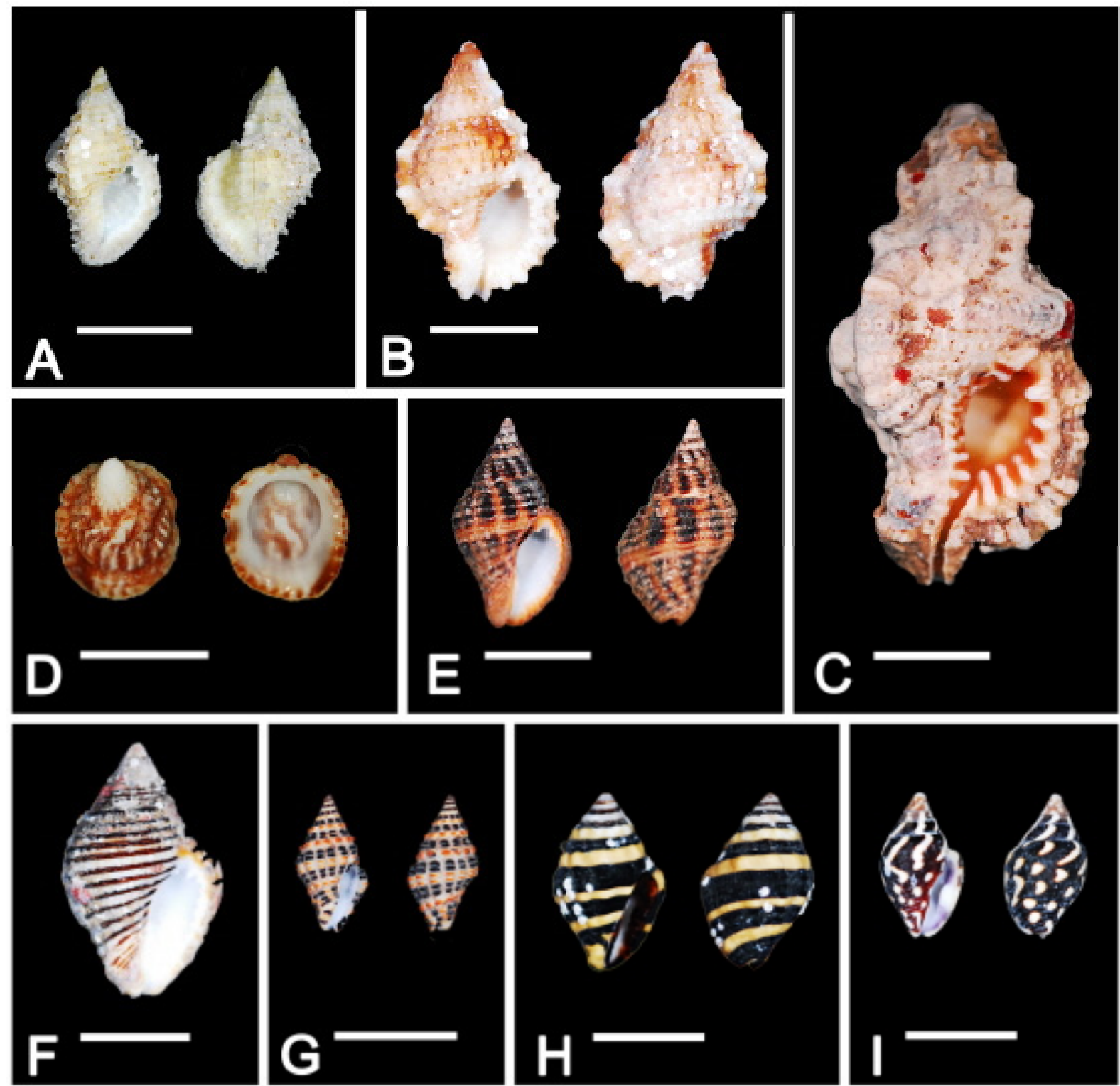

Plate 9: A, Cymatium mundum (scale bar = $10 \mathrm{~mm}$ ); B, Gyrineum natator (scale bar = $10 \mathrm{~mm}$ ); C, Cymatium nicobaricum (scale bar $=10 \mathrm{~mm}$ ); $\mathbf{D}$, Hipponix conicus (scale bar $=10 \mathrm{~mm}$ ); $\mathbf{E}$, Cantharus fumosus (scale bar $=10$ $\mathrm{mm}$ ); $\mathbf{F}$, Cantharus undosus (scale bar $=10 \mathrm{~mm}$ ); $\mathbf{G}$, Engina alveolata (scale bar $=10 \mathrm{~mm}$ ); $\mathbf{H}$, Engina mendicaria (scale bar $=10 \mathrm{~mm}$ ); I, Pyrene ocellata (scale bar $=10 \mathrm{~mm}$ ). 

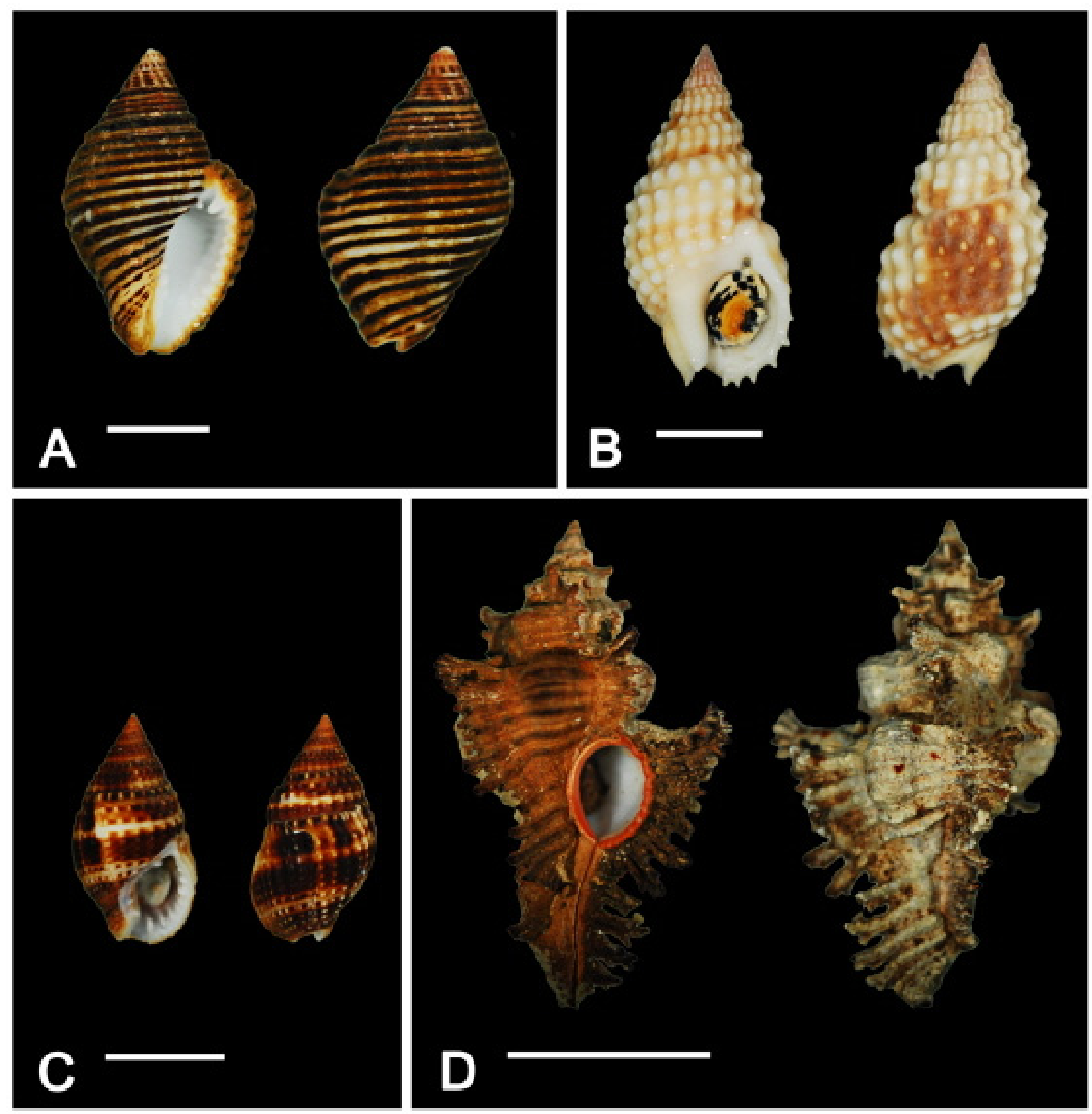

Plate 10: A, Latirolagena smaragdula (scale bar = $10 \mathrm{~mm}$ ); B, Nassarius papillosus (scale bar = $10 \mathrm{~mm}$ ); C, Nassarius margaritifer (scale bar = $10 \mathrm{~mm}$ ); $\mathbf{D}$, Chicoreus brunneus (scale bar $=30 \mathrm{~mm}$ ). 

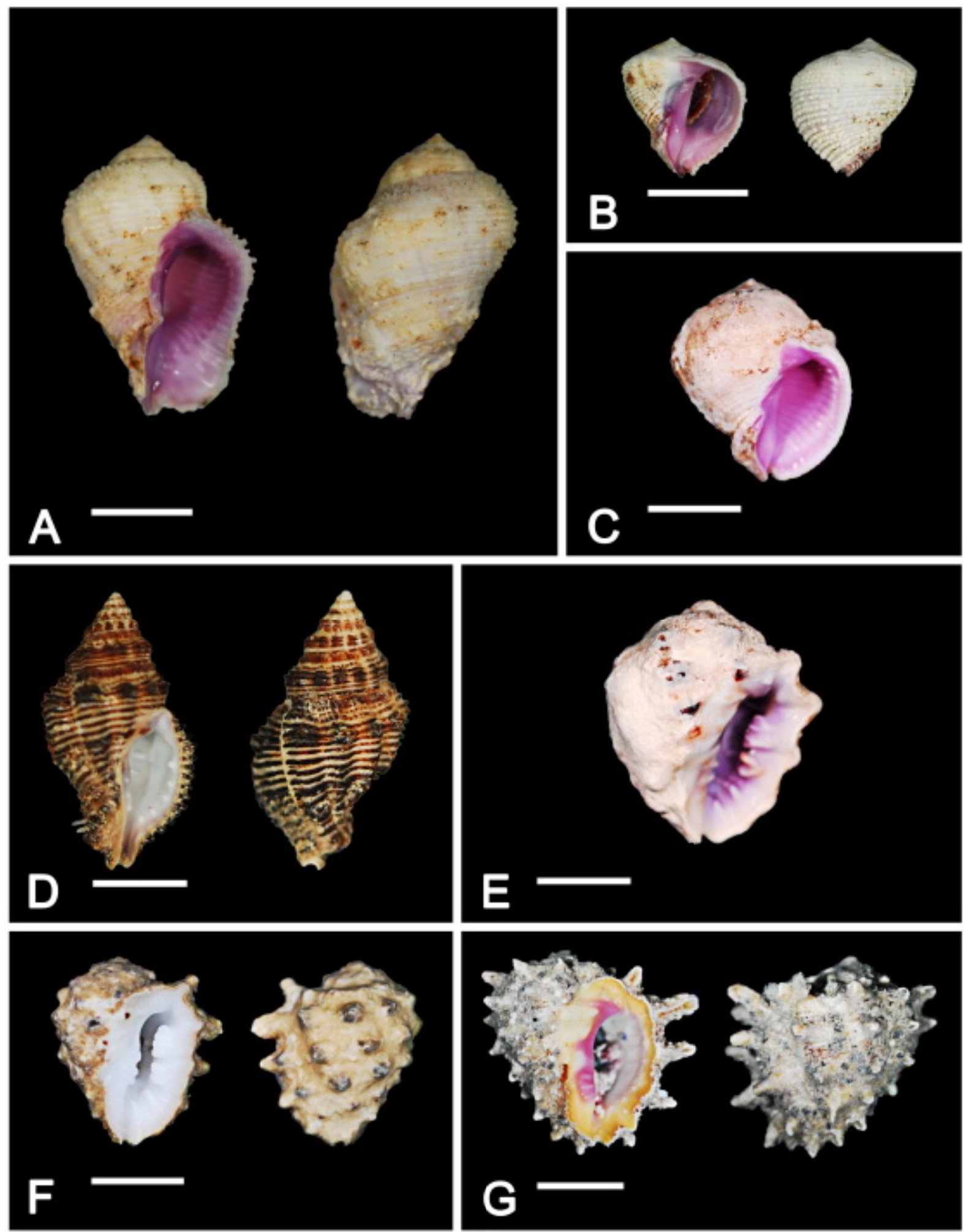

Plate 11: A, Coralliophila radula (scale bar = $10 \mathrm{~mm}$ ); B, Coralliophila squamulosa (scale bar $=10 \mathrm{~mm}$ ); C, Coralliophila vioalcea (scale bar = $10 \mathrm{~mm}$ ); D, Cronia margariticola (scale bar $=10 \mathrm{~mm}$ ); E, Drupa morum (scale bar = $10 \mathrm{~mm}) ; \mathbf{F}$, Drupa ricinus (scale bar $=10 \mathrm{~mm}$ ); G, Drupa rubusidaeus (scale bar $=10 \mathrm{~mm}$ ). 

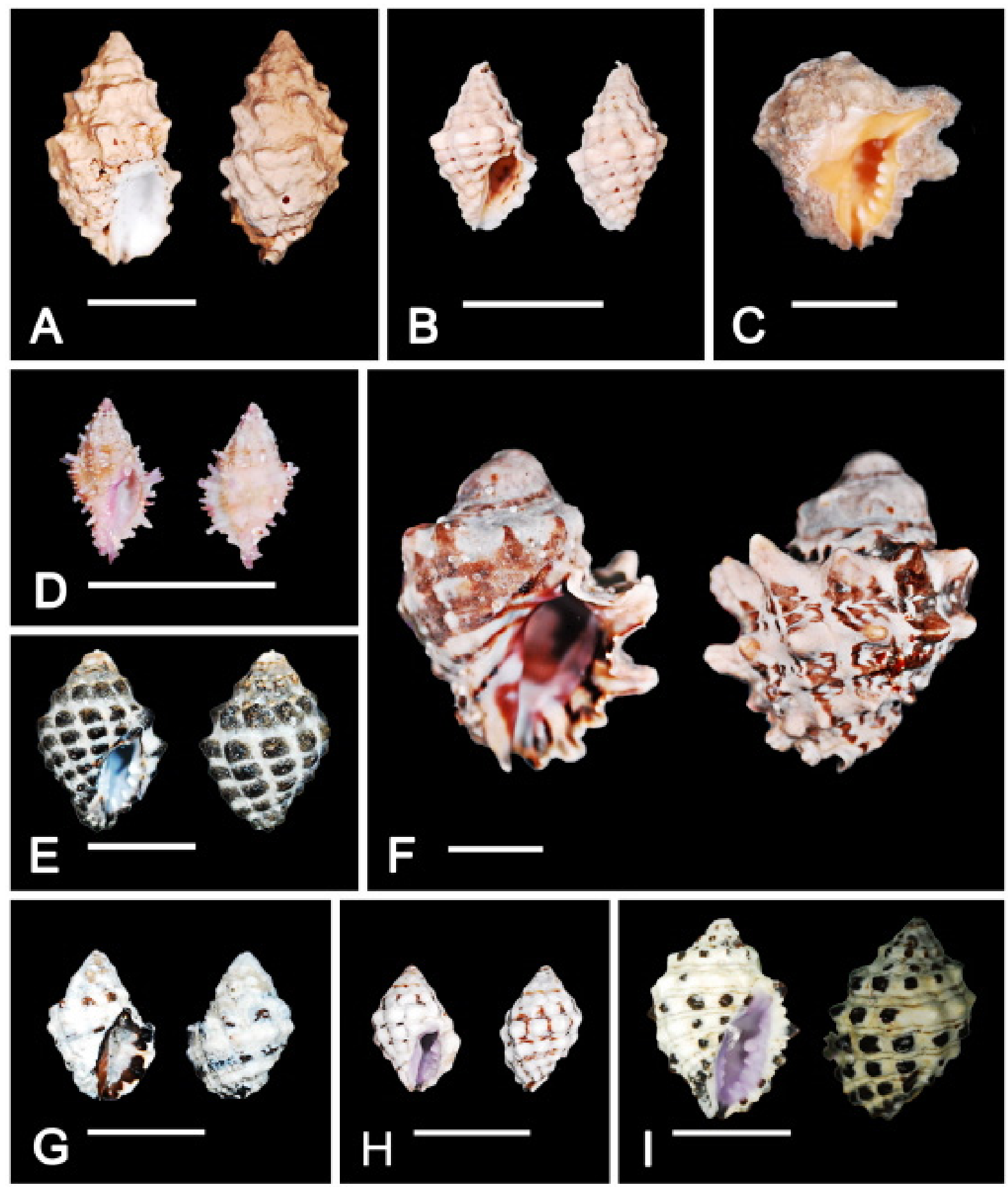

Plate 12: A, Drupells cornus (scale bar $=10 \mathrm{~mm}$ ); B, Drupella rugosa (scale bar $=10 \mathrm{~mm}$ ); C, Drupina grossularia (scale bar = $10 \mathrm{~mm}$ ); D, Habromorula ambrosia (scale bar = $10 \mathrm{~mm}$ ); E, Morula granulate (scale bar = $10 \mathrm{~mm}$ ); $\mathbf{F}$, Mancinella hippocastanus (scale bar $=10 \mathrm{~mm}$ ); G, Morula marginalba (scale bar $=10 \mathrm{~mm}$ ); $\mathbf{H}$, Morula nodicostata (scale bar $=10 \mathrm{~mm}) ;$ I, Morula uva (scale bar $=10 \mathrm{~mm}$ ). 

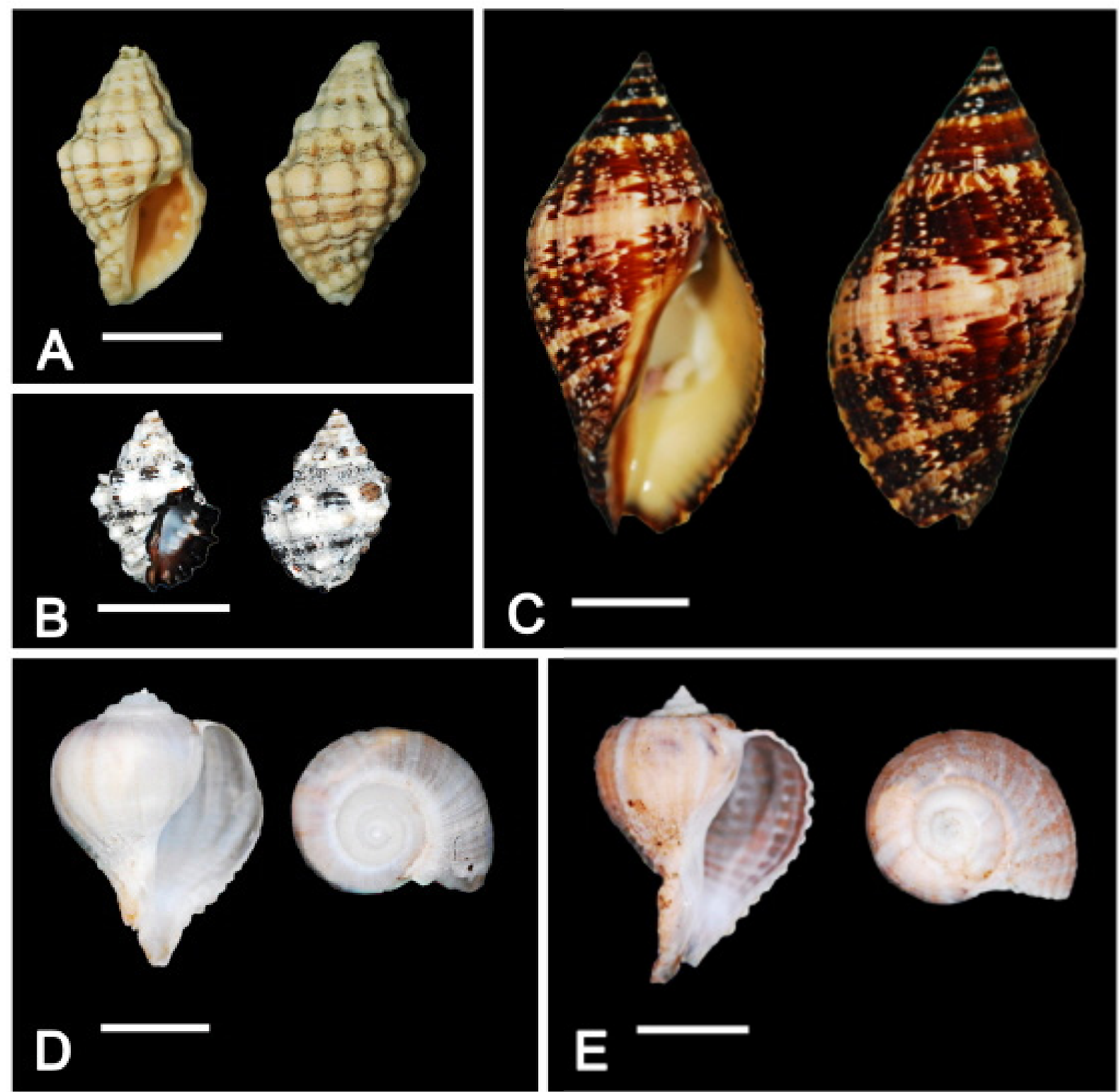

Plate 13: A, Muricodrupa fenestrate (scale bar = $10 \mathrm{~mm}$ ); B, Semiricinula marginatra (scale bar = $10 \mathrm{~mm}$ ); C, Nassa francolina (scale bar =10 mm); D, Rapa bulbiformis (scale bar =10 mm); E, Rapa rapa (scale bar $=10 \mathrm{~mm}$ ). 

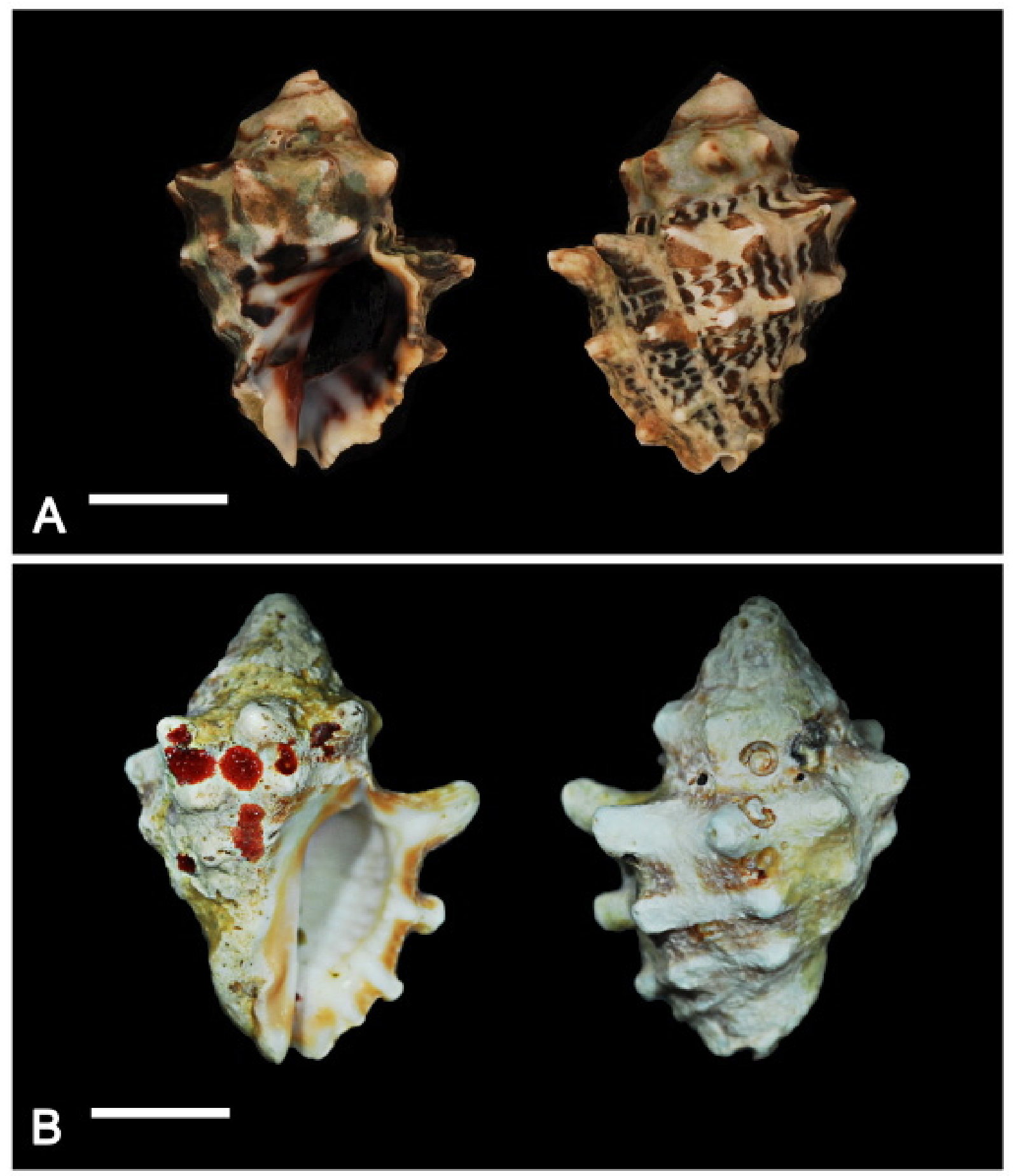

Plate 14: A, Thais aculeate (scale bar $=15 \mathrm{~mm})$; B, Thais armigera (scale bar $=15 \mathrm{~mm})$. 

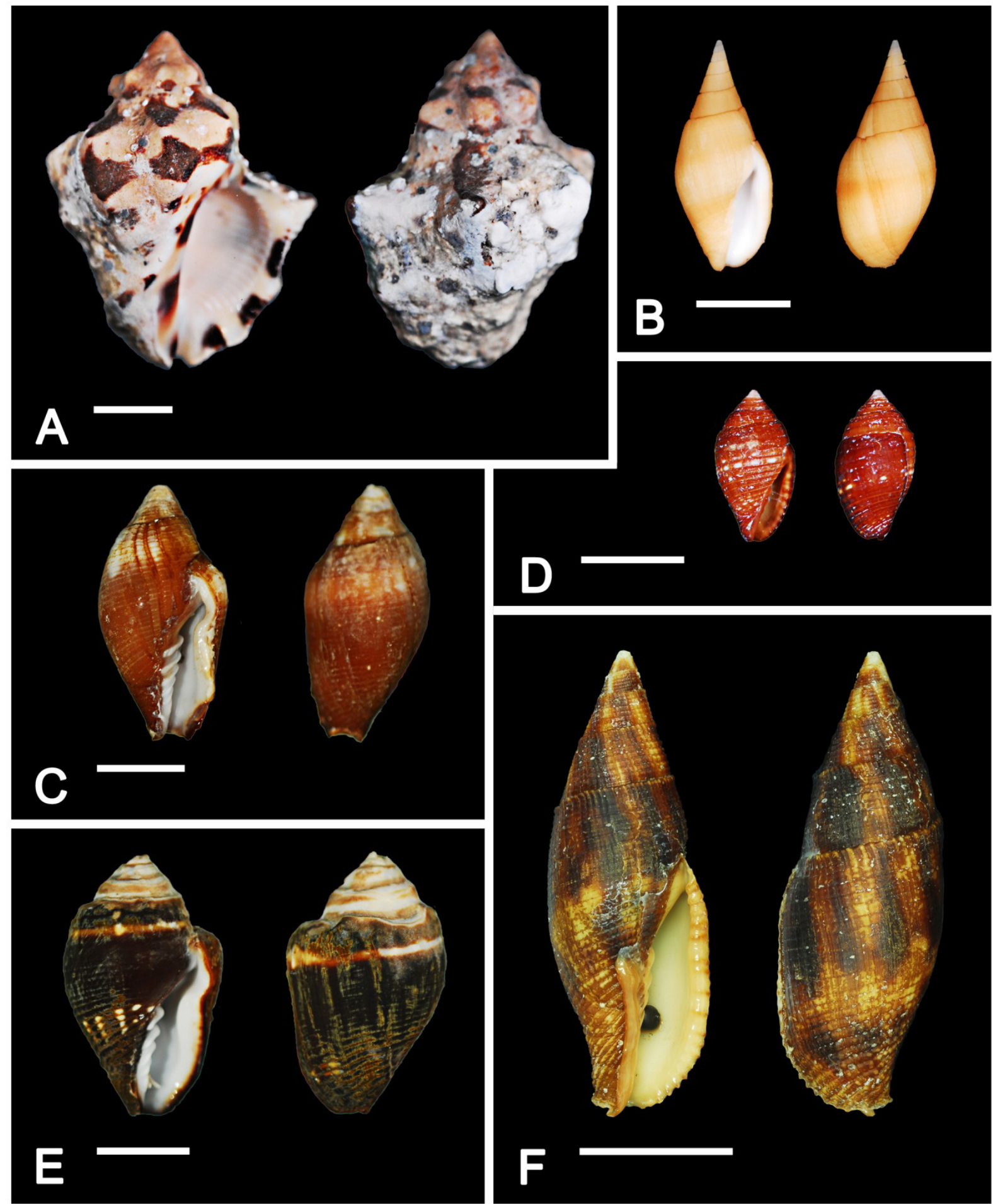

Plate 15: A, Thais tuberosa (scale bar $=10 \mathrm{~mm}$ ); B, Mitra acuminate (scale bar $=10 \mathrm{~mm}$ ); C, Mitra auriculoides (scale bar = $10 \mathrm{~mm}$ ); D, Mitra chrysalis (scale bar = $10 \mathrm{~mm}$ ); E, Mitra decurtata (scale bar = $10 \mathrm{~mm}$ ); F, Mitra eremitarum (scale bar $=20 \mathrm{~mm}$ ). 

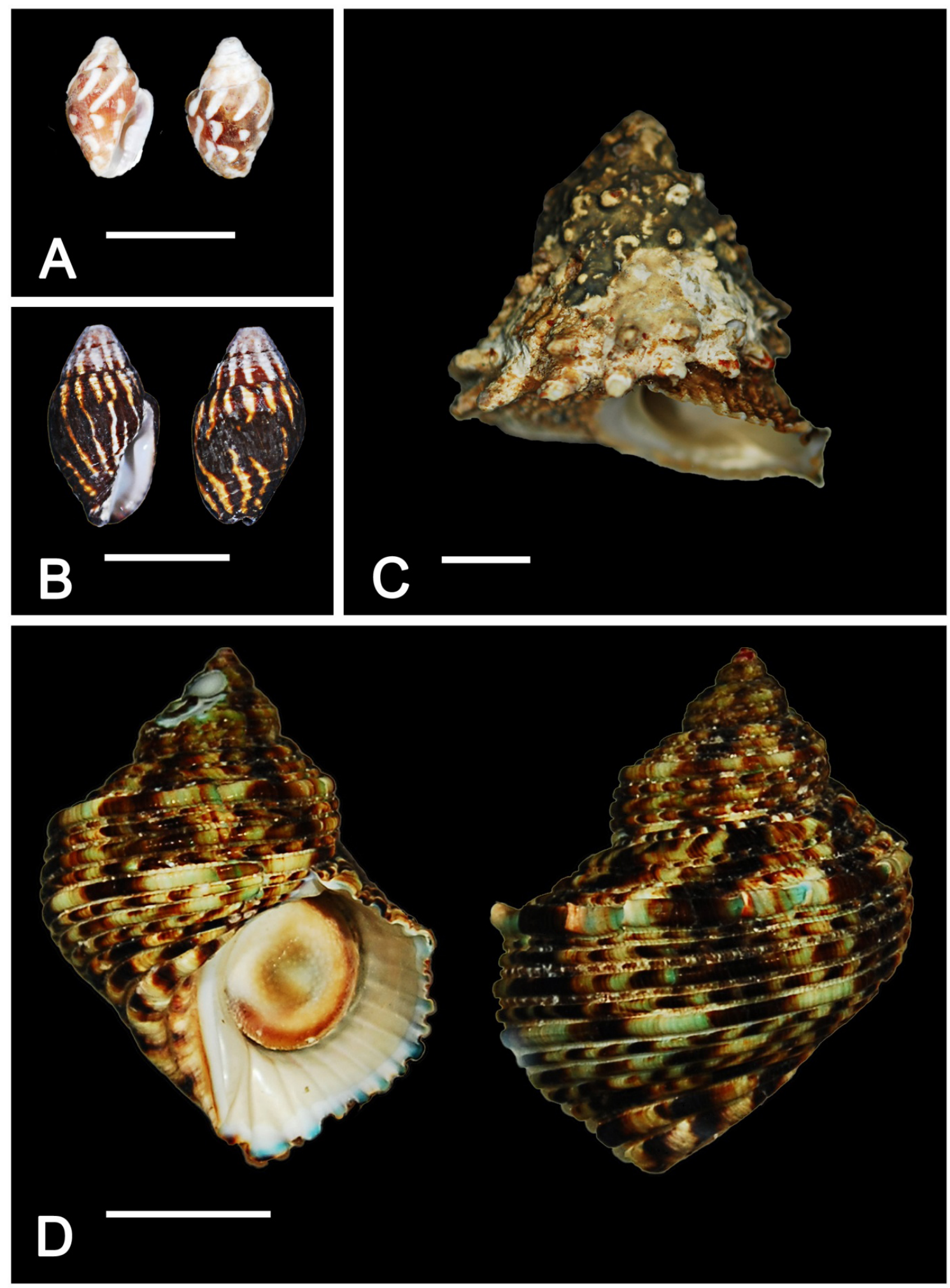

Plate 16: A, Mitra litterata (scale bar = $10 \mathrm{~mm}$ ); B, Mitra paupercula (scale bar = $10 \mathrm{~mm}$ ); C, Astralium rhodostoma (scale bar $=10 \mathrm{~mm}$ ); $\mathbf{D}$, Trubo argyrostomus (scale bar $=20 \mathrm{~mm}$ ). 

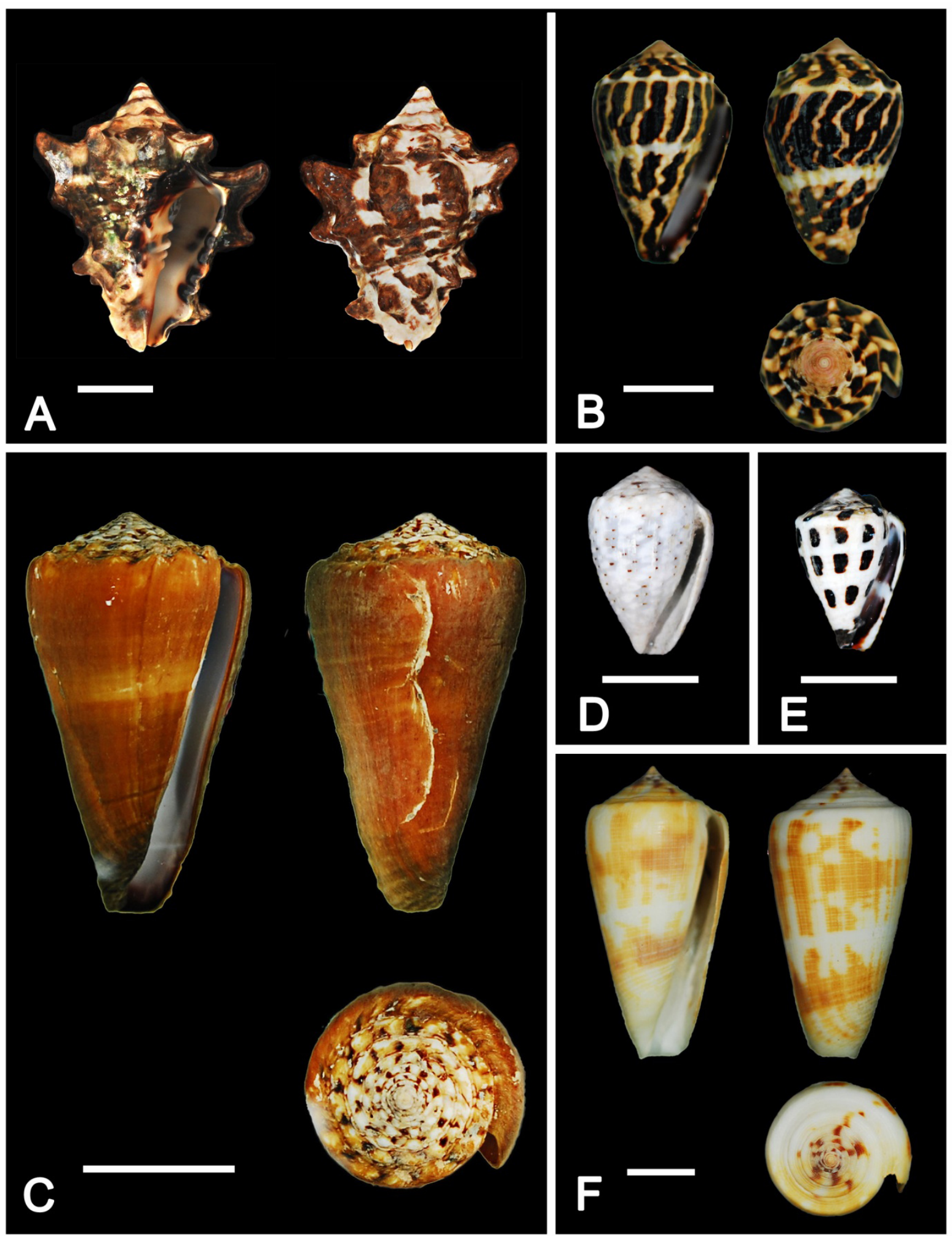

Plate 17: A, Vasum turbinellum (scale bar = $10 \mathrm{~mm}$ ); B, Conus chaldaeus (scale bar = $10 \mathrm{~mm}$ ); C, Conus distans (scale bar = $30 \mathrm{~mm}$ ); D, Conus coronatus (scale bar = $10 \mathrm{~mm}$ ); E, Conus ebraeus (scale bar = $10 \mathrm{~mm}$ ); $\mathbf{F}$, Conus giorossi (scale bar $=10 \mathrm{~mm}$ ). 

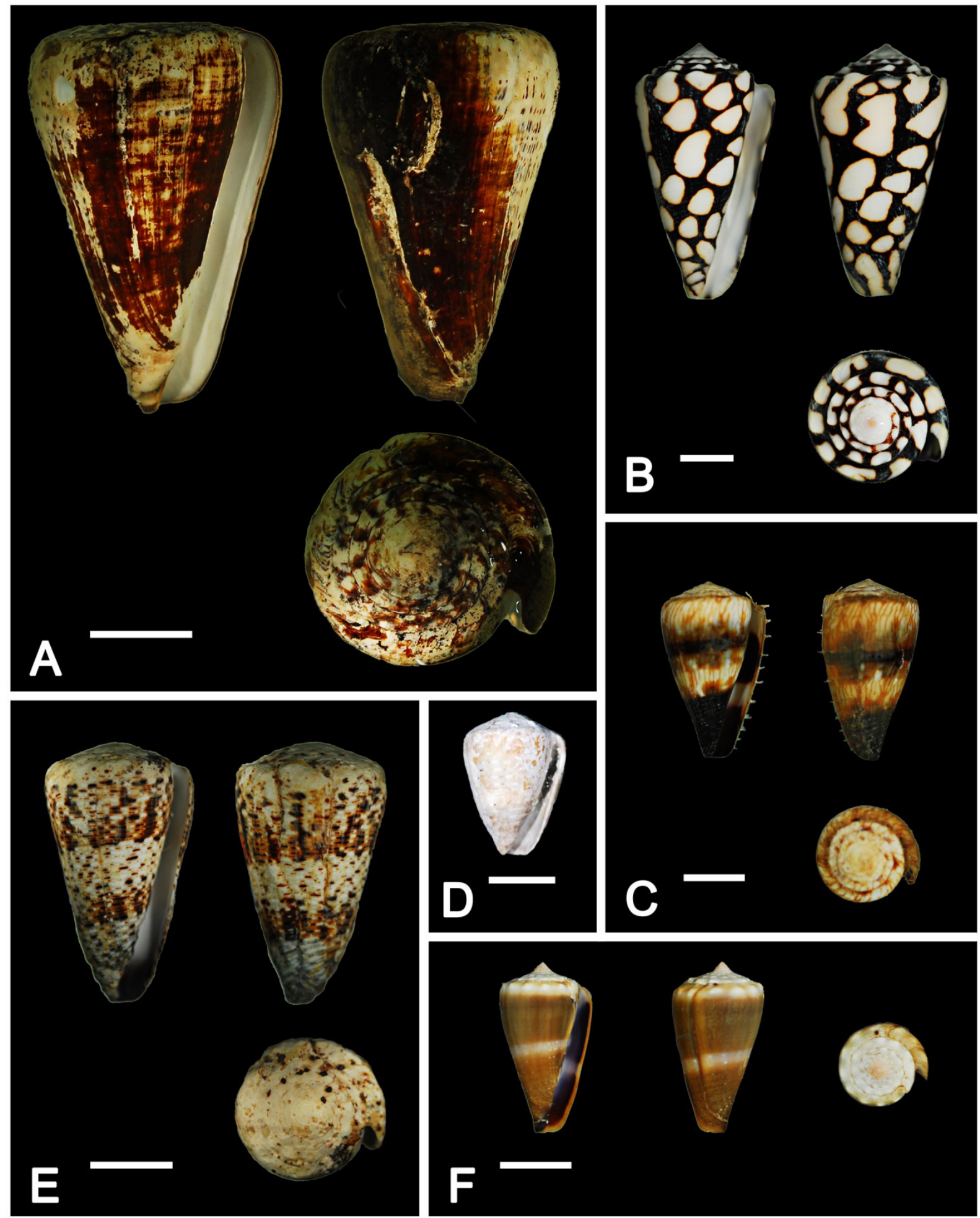

Plate 18: A, Conus leopardus (scale bar $=30 \mathrm{~mm}$ ); B, Conus marmoreus (scale bar $=10 \mathrm{~mm}$ ); C, Conus miles (scale bar $=10 \mathrm{~mm}$ ); $\mathbf{D}$, Conus miliaris (scale bar $=10 \mathrm{~mm}$ ); $\mathbf{E}$, Conus moncuri (scale bar $=20 \mathrm{~mm}$ ); $\mathbf{F}$, Conus moreleti (scale bar $=10 \mathrm{~mm}$ ). 

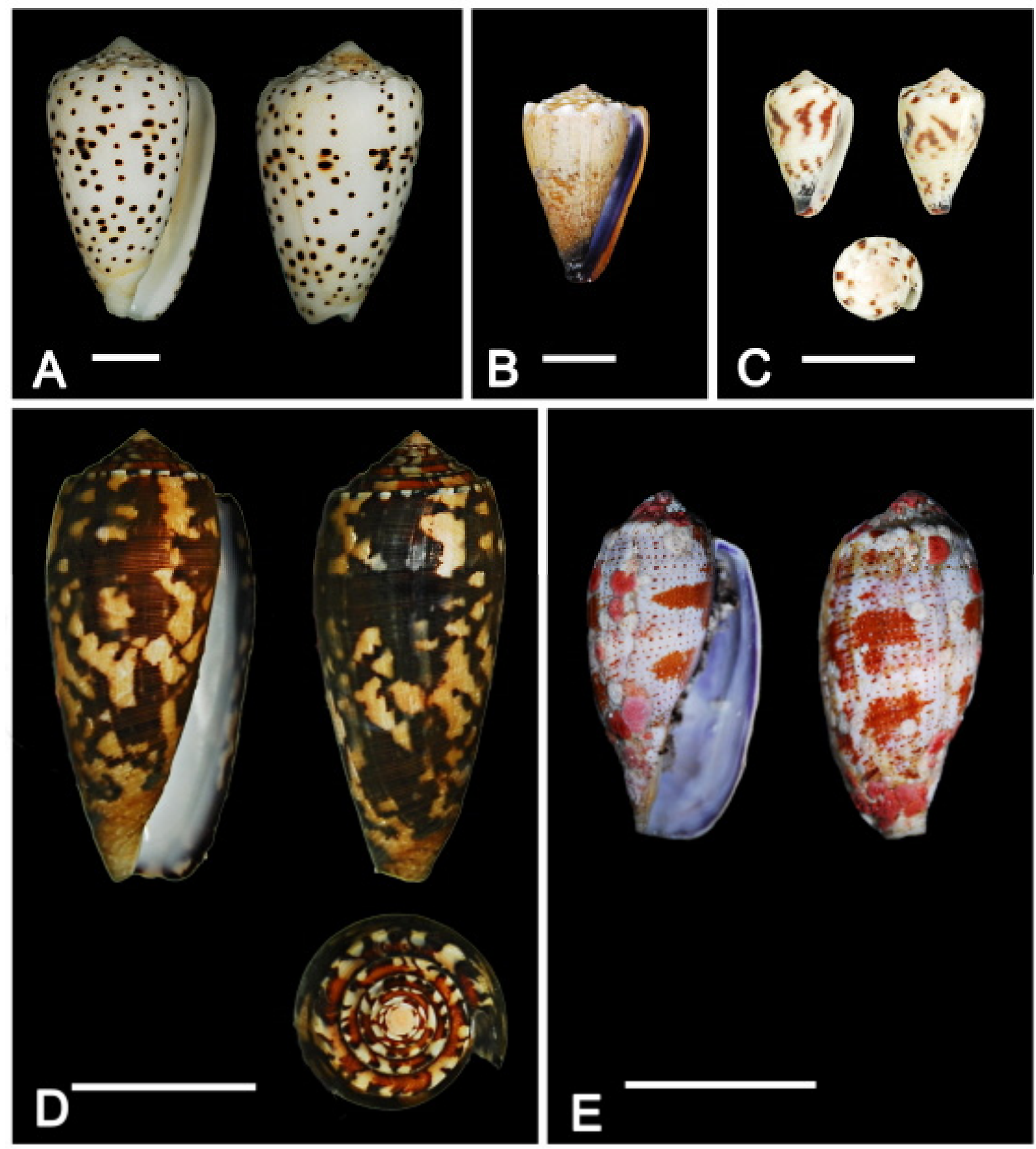

Plate 19: A, Conus pulicarius (scale bar = $10 \mathrm{~mm}$ ); B, Conus sanguinolentus (scale bar = $10 \mathrm{~mm}$ ); C, Conus sponsalis (scale bar = $10 \mathrm{~mm}$ ); D, Conus striatus (scale bar $=30 \mathrm{~mm}$ ); E, Conus tulipa (scale bar $=30 \mathrm{~mm}$ ). 


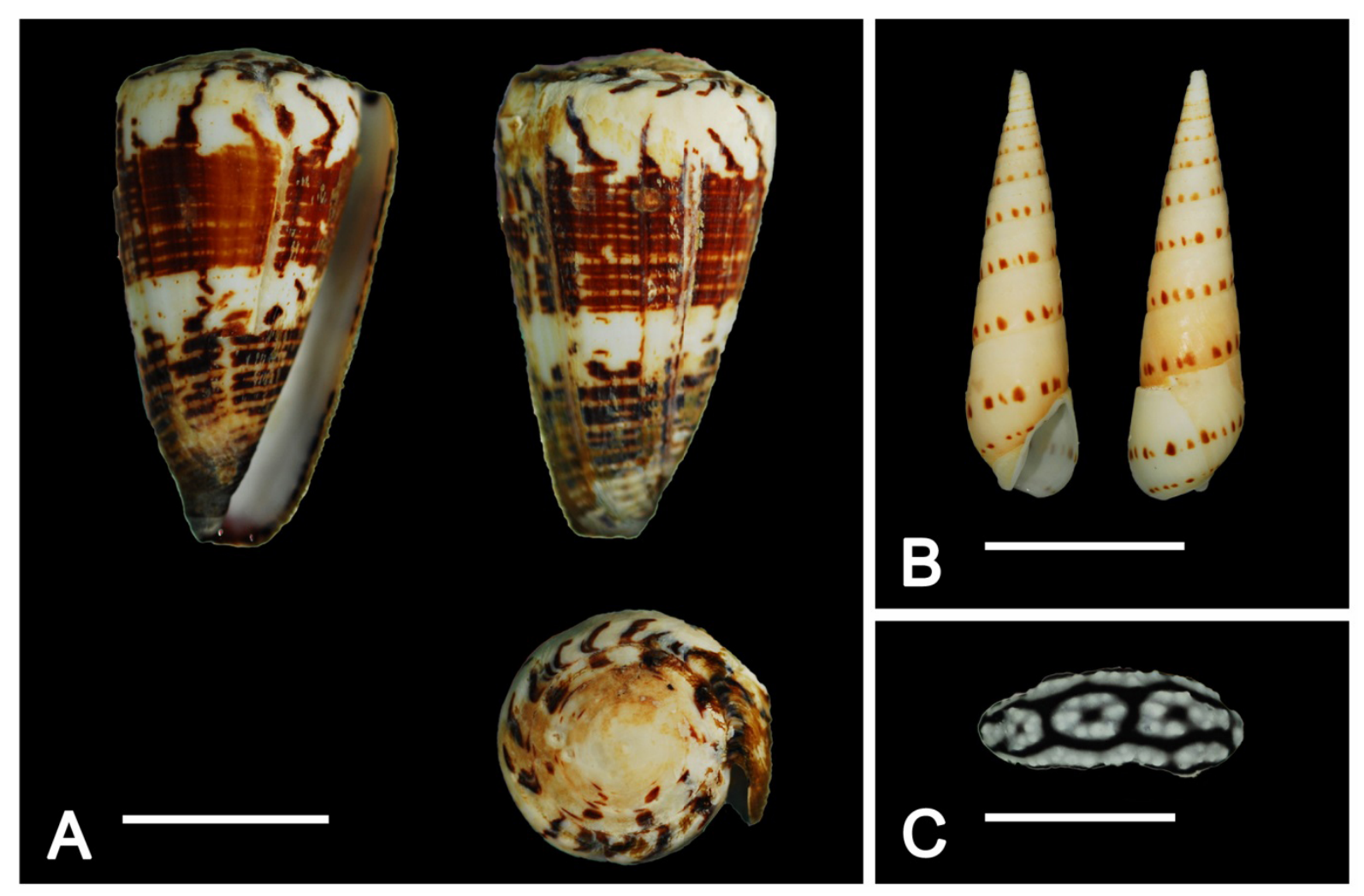

Plate 20: A, Conus vitulinus (scale bar $=20 \mathrm{~mm}$ ); B, Terebra feline (scale bar $=20 \mathrm{~mm}$ ); C, Phyllidiella pustulosa (scale bar $=10 \mathrm{~mm}$ ). 

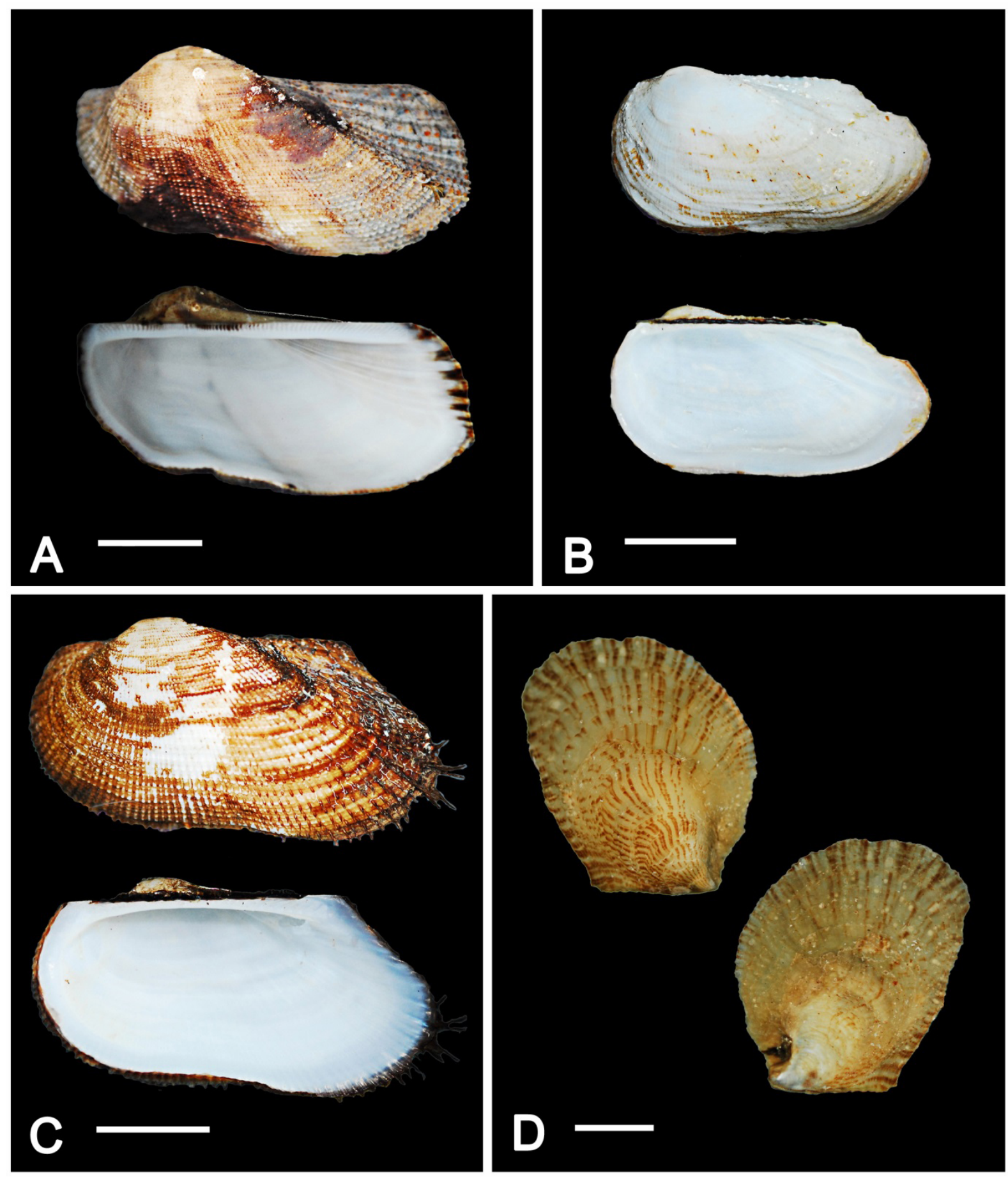

Plate 21: A, Arca avellana (scale bar $=10 \mathrm{~mm}$ ); B, Barbatia coma (scale bar $=10 \mathrm{~mm}$ ); C, Barbatia foliate (scale bar = $10 \mathrm{~mm}$ ); D, Isognomon perna (scale bar $=10 \mathrm{~mm}$ ). 

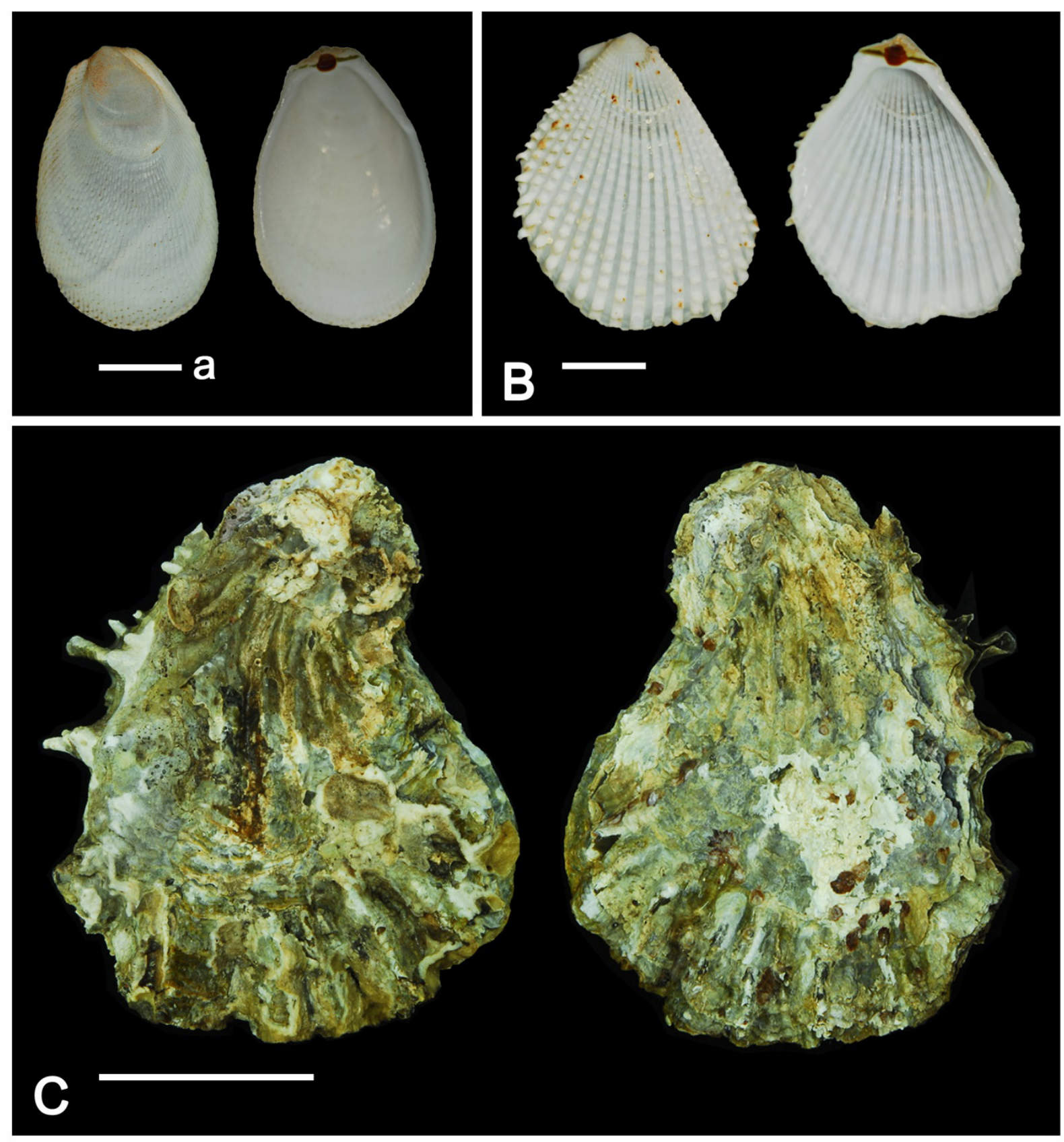

Plate 22: A, Ctenoides lischkei (scale bar $=10 \mathrm{~mm}$ ); B, Lima lima vulgaris (scale bar $=10 \mathrm{~mm}$ ); C, Hyotissa hyotis (scale bar $=80 \mathrm{~mm}$ ). 

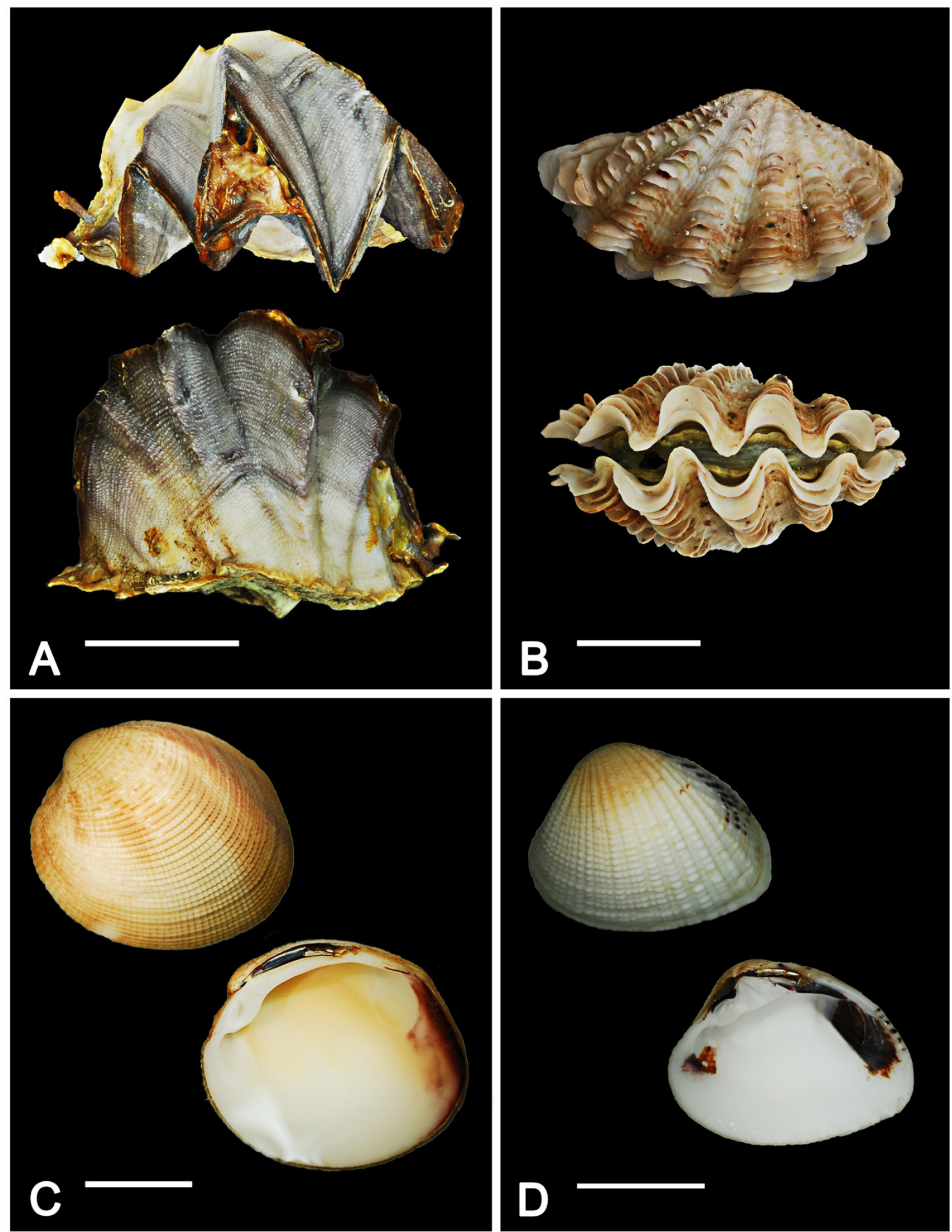

Plate 23: A, Lopha cristagalli (scale bar $=50 \mathrm{~mm}$ ); B, Tridacna maxima (scale bar $=30 \mathrm{~mm}$ ); C, Antigona puerperal (scale bar $=30 \mathrm{~mm}) ; \mathbf{D}$, Gafrarium menkei (scale bar $=30 \mathrm{~mm})$. 